Ann. Zootech., 1981, 30 (2), 209-248

\title{
Effets de la nature de l'amidon (blé ou maïs) et des protéines (poisson ou gluten) sur les facteurs de contrôle et le décours de l'évacuation gastrique d'un régime semi-purifié chez le Porc Applications de l'analyse multidimensionnelle et de la régression polynomiale
}

\author{
J.P. LAPLACE, Odile PONS *, J.C. CUBER, Chantal KABORE et P.A. VILLIERS \\ avec la collaboration technique de Brigitte Mourot et A. Van Hecke \\ I.N.R.A., Laboratoire de Physiologie de la Nutrition, \\ ' Laboratoire de Biométrie, \\ Centre de Recherches zootechniques, 78350 Jouy-en-Josas (France)
}

\begin{abstract}
Résumé
Douze porcs de race Large White, d'un poids vif moyen de $51,3 \mathrm{~kg}$, porteurs d'une canule gastrique permanente sont utilisés pour la mesure des contenus gastriques résiduels à divers temps $(30 \mathrm{mn}, 1,2,4$ et $7 \mathrm{~h})$ après un repas d'épreuve. Quatre régimes, formulés à 16 p. 100 de matières azotées, et résultant de la combinaison factorielle de 2 amidons purifiés (maïs ou blé) et de 2 sources protéiques (farine de poisson ou gluten de blé) sont distribués, après accoutumance, en 15 épreuves sur chaque porc ( 3 épreuves par temps de collecte ; 3 porcs par régime). Parallèlement, 2 régimes protéiprives à base d'amidon de maïs ou de blé sont utilisés sans accoutumance pour 2 épreuves au temps $2 \mathrm{~h}$ et 2 épreuves au temps $7 \mathrm{~h}$ chez chaque animal. Les quantités de matière fraîche, de matière sèche, d'amidon et d'azote restant dans l'estomac sont mesurées.

Une méthodologie élaborée d'analyse mathématique est mise en ouvre : elle est fondée sur l'analyse de variance multidimensionnelle pour tester les effets expérimentaux (amidon, protéine, porc), la décomposition polynomiale des effets pour mettre en évidence l'influence du temps, et la comparaison de profils.d'évacuation répondant à un ajustement polynomial des données. Les coefficients de ces polynômes font l'objat d'une interprétation physiologique.

Les variables contrôlées sont soumises à l'influence de la nature de l'amidon, de la source de protéines et de l'animal utilisé. Mais, il existe aussi des interactions telles que les effeis d'un amidon ou d'une protéine, évalués dans une situation donnée, n'autorisent aucune prévision de leurs effets respectifs dans d'autres associations. De plus, l'interaction des effets amidon et/ou protéine avec l'effet porc, en particulier à l'égard de l'azote, révèle l'originalité de chaque individu non seulement quant à sa production basale d'azote endogène mais quant à sa réponse à la nature des matériaux alimentaires qu'il ingère. L'influence du temps sur les observations est démontrée pour la matière sèche, l'amidon et l'azote. Une analyse des modifications du degré d'activation des facteurs de contrôle de l'évacuation est réalisée au travers des coefficients polynomiaux. Mais des niveaux très différents de ces facteurs peuvent conduire à des profils d'évacuation similaires, voire égaux. Les comparaisons
\end{abstract}


des profils d'évacuation obtenus pour chacune des variables contrôlées sont intégralement exprimées dans la figure 4 pour les régimes mis en œuvre. Enfin, l'évacuation gastrique n'apparaît pas comme un facieur de contrôle limitant de l'utilisation digestive des aliments.

\section{Introduction}

L'alimentation du porc comporte habituellement diverses céréales et sources de protéines. L'utilisation digestive de ces matières premières, ou de leur mélange en proportions variables, a fait l'objet de nombreuses évaluations. Parmi les facteurs de variation de cette utilisation, le rythme d'évacuation gastrique pourrait jouer un iôle non négligeable par l'intermédiaire du contrôle des quantités de matériaux soumises aux actions enzymatiques et de la cinétique d’apport au contact des surfaces absorbantes.

Bien que la nature de l'amidon et des protéines, et leurs traitements technologiques, soient a priori susceptibles de modifier le rythme d'évacuation gastrique, il existe peu d'études systématiques de ce problème. La digestibilité apparente de régimes à base de blé, sorgho, maïs ou orge, a été mesurée par KEYS \& DE BARTHE (1974) au niveau duodénal. Des informations sur la digestibilité de diverses variétés de blé (dur et tendre) ont été apportées par IVAN \& Farrel.L (1976) qui précisent en outre l'évolution des concentrations de lazote soluble et insoluble dans la matière sèche des contenus duodénaux. Dans le cas du maïs, soumis à divers procédés de conservation, Holmes, Bayley \& Horney (1974) décrivent la cinétique de passage de la matière sèche $(3,6,9$ et $12 \mathrm{~h}$ après le repas) et les quantités totales d'azote et d'amidon évacuées en 12 heures par l'estomac chez des porcs de $30 \mathrm{~kg}$ de poids vif. Des études cinétiques complètes du passage de la matière sèche, de l'azote et des glucides alimentaires ont été réalisées, également chez des porcs de $30 \mathrm{~kg}$ de poids vif (Low, Partridge \& Sambrook, 1978 ; Low, 1979 ; Sambrook, 1979). Mais la comparaison par ces auteurs d'un régime orge-blé-poisson à un régime purifié comportant un amidon de maïs, du saccharose, et selon les cas de la caséine ou du tourteau d'arachide, ne permet pas d'évaluer l'influence respective de lamidon ou de la protéine du régime sur l'évacuation gastrique. De même, le travail réalisé chez des porcs de $60 \mathrm{~kg}$ par ZEBRowSKa (1973 a) fournit des cinétiques de passage de la matière sèche et de l'azote lors d'ingestion de régimes dont les protéines proviennent selon les cas de caséine, de gluten, de tourteau de soja, ou de féverole. Mais ces aliments comportent à la fois de la pomme de terre et de l'amidon de blé avec 10 p. 100 de saccharose et 5 p. 100 de glucose.

Devant la complexité des régimes utilisés, et l'hétérogénéité des informations disponibles, il nous a paru utile de préciser l'influence éventuelle de la nature de l'amidon et des protéines sur l'évacuation gastrique chez le porc, en utilisant d'une part des régimes purifiés fondés sur la combinaison factorielle de 2 amidons et de 2 protéines et d'autre part la fistulation gastrique qui permet de garantir la vacuité de l'estomac avant le repas d'épreuve, et de limiter diverses interférences gênantes (azote endogène, pigments biliaires) dans les contenus duodénaux (CuBER, LAPLACE \& Villiers, 1980). 


\section{Matériel et méthodes}

Douze porcs de race Large White, mâles castrés, d'un poids vif de $51,3 \pm 0,6 \mathrm{~kg}$ (moyenne \pm écart-type de la moyenne) ont été utilisés. Sous anesthésie générale à l'halothane, on réalise une laparotomie sur la ligne médio-ventrale, entre l'appendice xyphoïde et l'ombilic. Une canule en vinyle (diamètres int./ext. 22-30 mm) est mise en place après gastrotomie à mi-hauteur de la grande courbure de l'estomac. Elle est extériorisée, par la plaie opératoire, juste en arrière de l'appendice xyphoïde.

\section{1. - Schéma expérimental et aliments}

Ces animaux sont affectés au hasard, à raison de 3 porcs par régime, à l'une ou l'autre des 4 combinaisons résultant du schéma factoriel $2 \times 2$. Ces régimes sont formulés manuellement sous deux contraintes essentielles : un taux de matières azotées totales de $16 \mathrm{p} .100$, et un taux de cellulose de 6 p. 100. La totalité des protéines provient selon le cas d'une farine de poisson ou de gluten de blé. Deux amidons purifiés sont utilisés, l'un de maïs, l'autre de blé. La cellulose est dans tous les cas une cellulose de bois purifiée. La composition centésimale des 4 aliments résultant de la combinaison des 2 sources de protéines et des 2 amidons est indiquée dans le tableau 1 , ainsi que les teneurs réelles en amidon et en azote déterminées sur 3 échantillons de chaque régime. Parallèlement, deux aliments protéiprives à base d'amidon purifié de maïs ou de blé ont été utilisés chez les mêmes animaux.

\section{2. - Alimentation et collecte des digesta}

Les porcs installés individuellement en cage à métabolisme sont habitués aux aliments expérimentaux (régimes à 16 p. 100 de matières azotées) durant 8 jours avant toute expérimentation. Ils reçoivent systématiquement 2 repas quotidiens à $9 \mathrm{~h}$ et $16 \mathrm{~h}$, apportant chacun $1 \mathrm{~kg}$ de farine fraîche diluée par $1,5 \mathrm{l}$ d'eau, soit $880 \mathrm{~g}$ de matière sèche (M.S.). Les quantités correspondantes d'amidon et d'azote ingérées lors du repas d'épreuve sont indiquées pour chaque régime dans le tableau 1. L'eau de boisson est apportée en totalité par l'eau de dilution de l'aliment, en l'absence de tout abreuvement complémentaire.

La collecte des contenus gastriques est pratiquée dans tous les cas à la suite du repas du matin. Avant le repas d'épreuve $(9 \mathrm{~h})$, on procède au contrôle de la vacuité gastrique : celle-ci est assurée par un abreuvement fictif de $500 \mathrm{ml}$ avec dérivation, par la canule gastrique ouverte, de l'eau ingérée entraînant les particules alimentaires résiduelles. Après ingestion du repas, une seule collecte des contenus gastriques résiduels est effectuée pour chaque jour d'épreuve : l'ouverture de la canule, selon les cas à l'un des temps ci-après, $30 \mathrm{mn}-1 \mathrm{~h}-2 \mathrm{~h}-4 \mathrm{~h}-7 \mathrm{~h}$ après le repas, autorise la collecte complète des contenus gastriques résiduels. Le porc reçoit alors un repas de remplacement équivalant à la quantité collectée, sur la base d'une teneur moyenne en M.S. des effluents gastriques de $30 \mathrm{p}$. 100. Lorsque la collecte a lieu au temps $7 \mathrm{~h}$ après le repas d'épreuve, le repas de remplacement est fourni en une fois en complément du second repas quotidien $(16 \mathrm{~h})$. Trois cinétiques complètes sont constituées chez chacun des 3 porcs affectés à chaque régime. En d'autres termes, pour un régime 


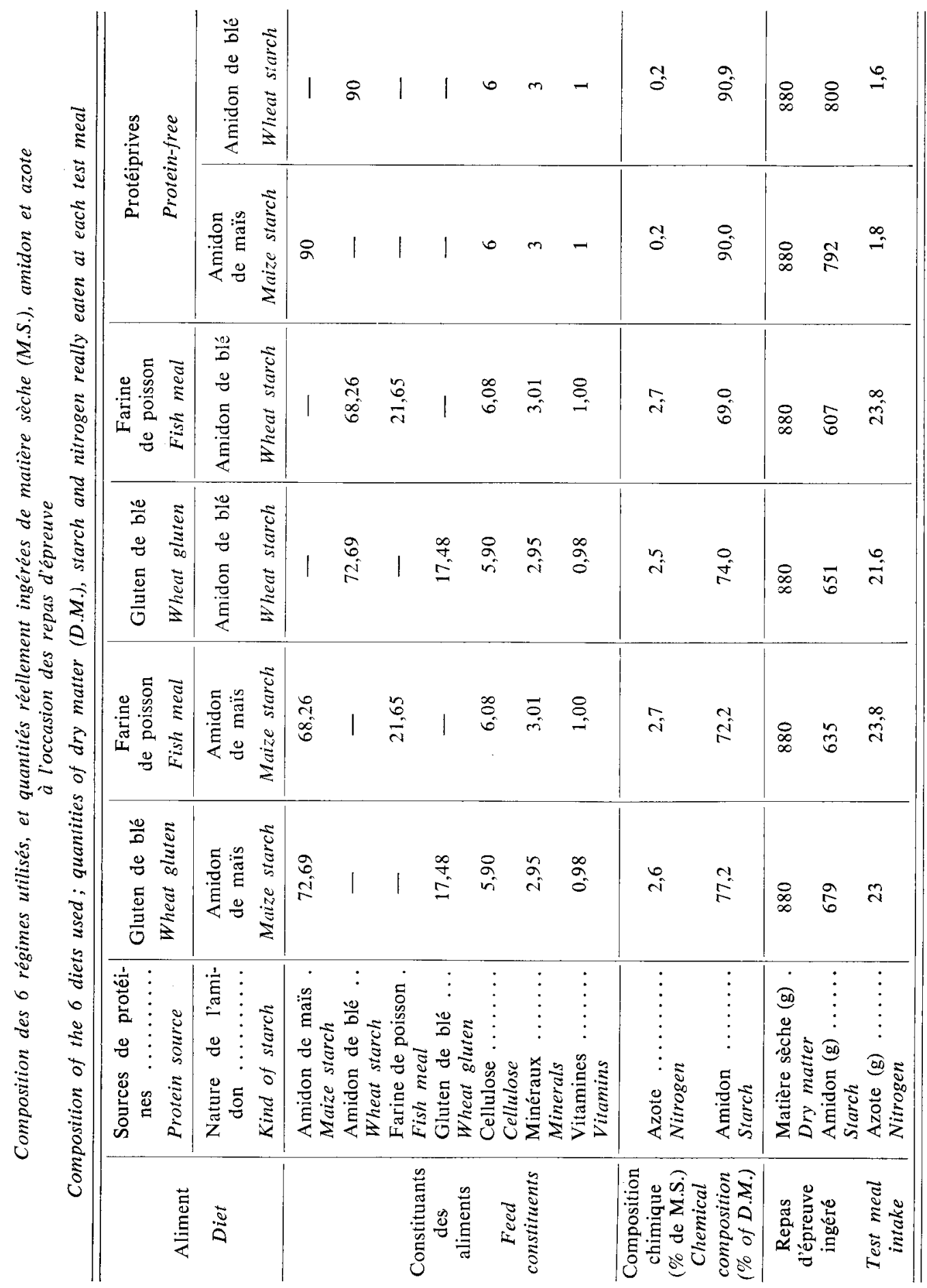


donné et pour chaque temps de mesure, 3 essais sont effectués chez chaque animal, soit 15 mesures pour un même porc ou encore un total de 9 mesures pour chaque instant d'observation et pour un régime donné.

Les régimes protéiprives n'ont fait l'objet de collectes qu'aux temps $2 \mathrm{~h}$ ou $7 \mathrm{~h}$ après le repas d'épreuve selon les jours. Les porcs ne sont pas accoutumés à ingérer ces aliments protéiprives qu'ils reçoivent seulement lors du repas du soir distribué à la veille d'une épreuve protéiprive.

\section{3. - Méthodes analytiques}

Les contenus gastriques résiduels sont pesés, homogénéisés vigoureusement et échantillonnés. Un premier échantillon d'environ $30 \mathrm{~g}$ est utilisé pour la détermination de la teneur en M.S. ( $24 \mathrm{~h}$ à l'étuve à $104^{\circ} \mathrm{C}$ ). Un second échantillon de même importance est prélevé puis lyophilisé en vue de la détermination de l'azote total (méthode Kjeldahl) et de l'amidon défini pour ce travail comme se référant au glucose et à tous les polymères alpha du glucose (dosage selon CuBEr \& LAPLACE, 1979). Ces diverses mesures permettent le calcul de la quantité totale de M.S. résiduelle dans l'estomac aux divers temps de collecte, ainsi que des quantités totales correspondantes d'azote et d'amidon.

Tous les résultats relatifs aux quantités de matières azotées sont calculés en grammes d'azote, tel que mesuré par la méthode Kjeldahl, et non en matières azotées totales (azote affecté du coefficient 6,25), en raison de la différence importante entre les 2 sources protéiques utilisées, et par analogie avec le mode d'expression usuel (Zebrowska, 1973 a ; Holmes, Bayley \& Horney, 1974 ; Low, 1979).

\section{4. - Présentation des résultats et analyse statistique}

\section{a) Description des données}

Les quantités de matière frâiche et de matière sèche ingérées étant identiques pour tous les régimes, les quantités résiduelles recueillies aux divers temps peuvent être décrites et comparées en valeur absolue. Par contre, du fait des variations des quantités d'amidon et d'azote effectivement ingérées (tabl. 1), les quantités résiduelles de ces deux composants ne peuvent être comparées que sur la base de la fraction (en p. 100) de la quantité ingérée recueillie à tel ou tel temps post-prandial, et relativement aux quantités résiduelles de matière fraîche ou sèche exprimées selon le même mode.

Les résultats moyens présentés sous forme graphique (régimes azotés) ou numérique (régimes protéiprives) avec indication des écarts types des moyennes, font l'objet de comparaisons deux à deux par un test $\mathbf{T}$ de Student.

\section{b) Analyses de variance : effets amidon, protéine, porc}

Au-delà de la simple description, l'analyse des effets susceptibles de rendre compte des différences de profil d'évacuation gastrique (dans le cadre du schéma factoriel, régimes azotés) a été conduite sur les quantités évacuées ( $Q$ ingérée - $Q$ résiduelle) exprimées en grammes. Ces observations de la variable «quantité évacuée », 
notée $Y$, sont obtenues successivement aux instants $t_{1}, t_{2}, \ldots, t_{5}(30 \mathrm{~min}, 1 \mathrm{~h}, \ldots, 7 \mathrm{~h})$ sur un même animal. Elles ne sont donc pas indépendantes, aussi le temps ne peut-il être considéré comme un facteur explicatif de l'analyse de variance au même titre que les autres facteurs (nature de l'amidon, source de protéines, animal).

Un modèle d'analyse de variance multidimensionnelle (KRISHNAIAH, 1969) prend en compte cette dépendance en étudiant l'ensemble des variables y $\left(t_{1}\right), \ldots$, y $\left(t_{5}\right)$. Pour chacune des variables y $(t),\left(t=t_{1}, \ldots, t_{5}\right)$, l'observation pour le ieme amidon, la jeme protéine, le keme porc et la leme cinétique est décrite par :

$$
Y_{i j k l}(t)=\mu(t)+a_{i}(t)+b_{j}(t)+c_{k}(t)+(a b)_{i j}(t)+(a c)_{i k}(t)+(b c)_{j k}(t)+
$$
$(a b c)_{i j k i}(t)+\Sigma_{i j k l}(t)$

$\mu(t)$ est la moyenne générale des $Y_{i j k l}(t)$ quand les indices $i, j, k, 1$ varient ;

$a_{j}(t)$ est l'effet du ieme amidon, au temps $t$, estimé par l'écart à $\mu(t)$ de la moyenne des $Y_{i j k l}(t)$ quand $j, k, 1$ varient;

$b_{j}(t)$ est l'effet de la jeme proteine, au temps $t$, estimé par l'écart à $\mu(t)$ de la moyenne des $Y_{i j k l}(t)$ quand $i, k, 1$ varient; varient ;

$c_{\mathrm{k}}(\mathrm{t})$ est l'effet du keme porc, au temps t, estimé de la même façon quand $\mathrm{i}, \mathrm{j}, 1$

Les $(a b)_{i j}(t),(a c)_{i k}(t),(b c)_{j k}(t)$ et $(a b c)_{i j k}(t)$ sont les interactions au temps $t$ des effets indiqués;

$\Sigma_{\mathrm{ijkl}}(\mathrm{t})$ est la résiduelle.

Les estimateurs des effets et interactions ainsi définis sont les mêmes que si l'on effectuait séparément des estimations sur y $\left(\mathrm{t}_{1}\right), \mathrm{y}\left(\mathrm{t}_{\mathrm{z}}\right)$, .. et y $\left(\mathrm{t}_{\overline{\mathrm{a}}}\right)$.

En pratique, cinq analyses de variance distinctes ont été classiquement conduites pour tester les effets (amidon, protéine, porc) et leurs interactions sur les cinq groupes de données (recueillies respectivement aux temps $30 \mathrm{mn}, 1 \mathrm{~h}, 2 \mathrm{~h}, 4 \mathrm{~h}$ et $7 \mathrm{~h}$ ) traités séparément. Puis une analyse de variance multidimensionnelle, selon le modèle exposé ci-dessus, a été conduite sur l'ensemble du corpus de données recueillies pour tous les régimes à tous les instants d'observation.

Les paramètres du modèle multidimensionnel correspondent à l'ensemble, constituant la matrice $0_{1}$, des effets des cinq modèles distincts. Ainsi pour l'effet amidon par exemple, cet effet $a_{1}$ est l'ensemble $\left[a_{i}\left(t_{1}\right), a_{i}\left(t_{2}\right), \ldots, a_{i}\left(t_{5}\right)\right]$. Du fait de la dépendance des $Y(t)$, les $a_{i}(t)$ sont dépendants et un test de l'effet amidon, global sur les cinq instants dobservation, ne peut être conduit par un test de Fisher. Parmi les tests utilisables, ceux de Roy, de Wilks \& de Lawley-Hotteling (Krishnaiah, 1969) ont été calculés pour nos observations. Alors que le test de Fisher pour une seule variable y est fondé sur la résiduelle $\varepsilon$ des modèles étudiés, les tests sont ici formés, en présence de cinq variables $\mathrm{Y}(\mathrm{t})$, à partir des matrices résiduelles,

$$
\left[\left(\Sigma_{\mathrm{ijkl}}\left(\mathrm{t}_{1}\right), \Sigma_{\mathrm{ijkl}}\left(\mathrm{t}_{2}\right), \ldots, \Sigma_{\mathrm{ijkl}}\left(\mathrm{t}_{5}\right)\right)_{\mathrm{ijkl}}\right]
$$

dont ils étudient différentes fonctions des valeurs propres. 
c) Mise en évidence de l'effet du temps; décomposition polynomiale des effets expérimentaux

Un ajustement par un polynôme de degré maximum (degré 4 pour 5 instants d'observation) a été effectué pour chacun des effets et interactions du modèle d'analyse de variance multidimensionnel (selon PotThoff \& RoY, 1964). A partir de la matrice $0_{1}$ des paramètres de l'analyse de variance multidimensionnelle, on établit une matrice $\theta_{2}$ constituée de l'ensemble des coefficients polynomiaux résultant des ajustements effectués pour chacun des effets et interactions du modèle, c'est-à-dire pour chaque terme de $\theta_{1}$. Sur ces coefficients composant la matrice $\theta_{2}$, on procède à un test de nullité des coefficients polynomiaux du degré le plus élevé. Ce test de nullité a été effectué par les trois méthodes de Roy, Wilks \& LAWLEY-HotTeling: (Krishnaiah, 1969); il permet de déterminer le degré minimum de la courbe qui fournit le meilleur ajustement pour tous les termes de lanalyse de variance dans le cas de chacune des variables étudiées (matière fraîche, matière sèche, amidon et azote évacués). L’influence du temps peut alors être approchée au travers des coefficients des polynômes ainsi définis.

d) Comparaison des courbes moyennes pour chaque couple de traitement amidonprotéine

Les analyses de variance effectuées, tant pour chaque instant d'observation que sur l'ensemble du corpus de données (modèle multidimensionnel), révélant l'existence d'interactions significatives, une comparaison des profils d'évacuation gastrique selon les régimes (4 couples amidon-protéine) est difficile à mettre en œuvre. Compte tenu du degré du polynôme retenu lors de l'étape précédente pour chacune des variables étudiées, un ajustement moyen pour les (3 pores $\times 3$ cinétiques) a été effectué par régression polynomiale pour chacun des 4 couples de traitement (i.e. régime ou combinaison d'un amidon et d'une protéine). La comparaison de ces courbes moyennes est alors basée sur celle des coefficients polynomiaux des ajustements moyens (*). Pour une variable donnée, la comparaison peut prendre en compte 2,3 ou 4 des courbes moyennes représentatives de chacun des 4 régimes. Les régimes sont indicés $r(r \leqslant 4)$, cet indice équivalant au groupe d'indices ij dans le modèle multidimensionnel puisque chaque régime équivaut à un couple amidon-protéine. Quel que soit le nombre de courbes comparées, on procède selon les 3 étapes ci-après, en définissant $\mathrm{I}=(1, \ldots, 4)$ comme un ensemble d'indices choisis parmi 1 à 4 selon les régimes mis en comparaison.

$\alpha$ - On établit pour chacune des variables étudiées les quatre courbes différentes initiales, répondant dans l'hypothèse d'un polynôme de degré 3 à l'écriture.

$$
a_{r}+b_{r} t+c_{r} t^{2}-d_{r} t^{3}
$$

On dispose pour chacune des comparaisons de courbes (correspondant à un régime) d'une valeur dite $R_{3}$ de la résiduelle, soit :

$$
R_{3}=\Sigma_{r} \in_{\mathrm{I}} \Sigma_{\mathrm{k} 1}\left(\mathrm{Y}_{\mathrm{rkl} 1}-\left(\mathrm{a}_{\mathrm{r}}+\mathrm{b}_{\mathrm{r}} \mathrm{t}+\mathrm{c}_{\mathrm{r}} \mathrm{t}^{2}+\mathrm{d}_{\mathrm{r}} \mathrm{t}^{3}\right)\right)^{2}
$$

(*) Les coefficients polynomiaux de ces ajustements moyens n'ont rien de commun avec les coefficients polynomiaux constituant la matrice $\theta_{2}$ lors de l'étape précédente de décomposition polynomiale des effets. Il s'agit cette fois d'un ajustement global sur les données ne différenciant plus les termes de l'analyse de variance. 
$\beta$ - Pour la variable considérée et pour chaque courbe initiale on construit quatre courbes parallèles ne différant que par le terme constant du polynôme soit :

$$
a_{\mathbf{r}}+b t+c t^{2}+d t^{3}
$$
sons :

On définit une valeur dite $R_{2}$ comme la résiduelle pour chacune des comparai-

$$
\mathrm{R}_{2}=\Sigma_{\mathrm{r}} \epsilon_{\mathrm{I}} \Sigma_{\mathrm{kl}}\left(\mathrm{Y}_{\mathrm{rk} 1}-\left(\mathrm{a}_{\mathrm{r}}+\mathrm{bt}+\mathrm{ct}^{2}+\mathrm{dt}^{3}\right)\right)^{2}
$$

$\gamma$ - On construit enfin une courbe commune répondant au polynôme :

$$
a+b t+c t^{2}+d t^{3}
$$

On définit la valeur dite $R_{1}$ comme la résiduelle pour chacune des comparaisons :

$$
\mathrm{R}_{1}=\Sigma_{\mathrm{r}} \in_{\mathrm{I}} \Sigma_{\mathrm{k} i}\left(\mathrm{Y}_{\mathrm{rkl}}-\left(\mathrm{a}+\mathrm{bt}+\mathrm{ct}^{2}+\mathrm{dt}^{3}\right)\right)^{2}
$$

A partir de ces éléments, deux tests sont mis en œuvre :

- Un test d'égalité :

$$
\frac{\mathrm{R}_{1}-\mathrm{R}_{3} / \mathrm{ddl}}{\mathrm{R}_{3} / \mathrm{ddl}}
$$

ddl : nombre de degrés de liberté.

- Un test de parallélisme :

$$
\frac{\mathrm{R}_{2}-\mathrm{R}_{3} / \mathrm{ddl}}{\mathrm{R}_{3} / \mathrm{ddl}}
$$

Le test d'égalité teste l'écart de telle ou telle courbe initiale $(\alpha)$ par rapport à la courbe commune $(\gamma)$, reconnaissant ainsi l' "originalité » ou la non originalité du profil d'évacuation gastrique (exprimé sous sa forme polynomiale) pour telle variable étudiée lors d'ingestion de tel ou tel régime par rapport à un profil moyen d'évacuation.

Le test de parallélisme teste l'écart de telle ou telle courbe initiale $(\alpha)$ par rapport à la courbe correspondante $(\beta)$ reconnaissant ainsi la similitude à l'ordonnée à l'origine près (le parallélisme) ou le non parallélisme des profils d'évacuation gastrique (sous leur forme polynomiale) enregistrés pour telle variable étudiée, dans la ou les situations (régimes) mises en comparaison.

\section{e) Cas des régimes protéiprives}

Pour ce qui concerne les régimes protéiprives, seules ont été réalisées les étapes d'analyses de variance, soit analyses séparées pour les données relatives à chacun des deux instants d'observation, puis analyse de variance multidimensionnelle appliquée à l'ensemble des observations effectuées après ingestion de régimes protéiprives. Dans ce dernier cas, le modèle multidimensionnel, les effets étudiés et leurs interactions sont analogues à ce qui a été exposé précédemment pour les régimes azotés. Etant donné le nombre plus restreint de données, la décomposition polynomiale des effets et la comparaison de courbes moyennes n'ont pas été réalisées pour les régimes protéiprives. 


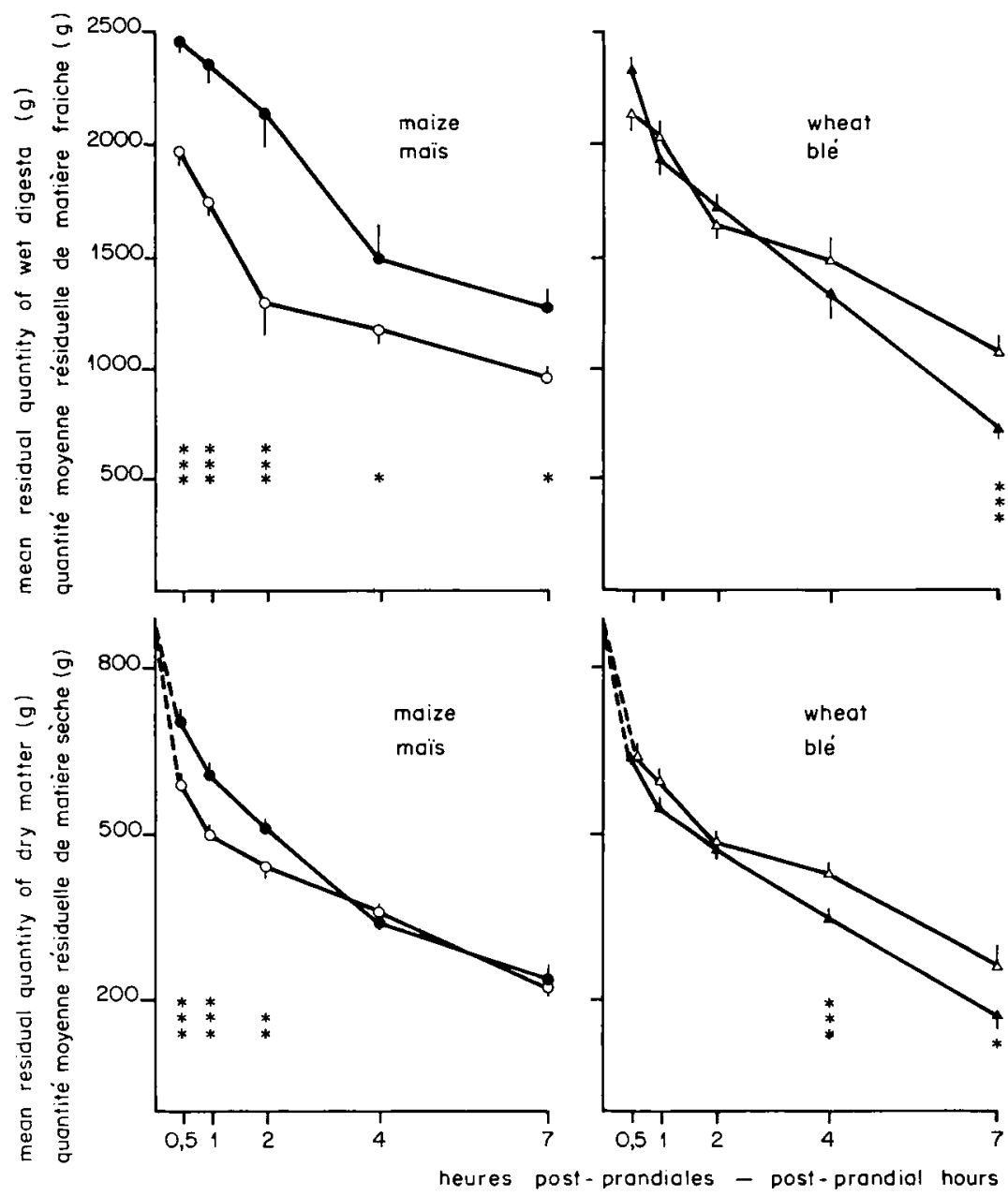

FIG. 1

Quantités moyennes (et écarts types des moyennes), résiduelles dans l'estomac (en g), de matière fraîche et de matière sèche après un repas d'épreuve (ingéré frais $2500 \mathrm{~g}$, sec $880 \mathrm{~g}$ ) selon la nature de l'amidon purifié et la source de protéines entrant dans la composition du régime. Les astérisques expriment le résultat d'un test $t$ de Student entre les données obtenues à chaque temps de collecte :

$$
*(p<0,10) ; * * * 0,05) ; * * *(p<0,01)
$$

Mean (士 SEM) residual quantities in the stomach $(g)$ of wet digesta and dry matter after a test meal (intake : $2500 \mathrm{~g}$ wet, $880 \mathrm{~g}$ dry) according to the kind of purified starch and to the protein source. Asterisks show the results of a Student's $t$ test between the data at each collection time :

$*(p<0.10) ; * *(p<0.05) ; * * *(p<0.01)$

Régimes maïs (Maize diets)

- Farine de poisson (Fish meal)

$\mathrm{O}-$ Gluten de blé (Wheat gluten)

Régimes blé (Wheat diets)

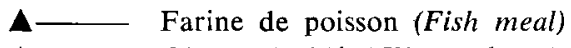

$\triangle \longrightarrow$ Gluten de blé (Wheat gluten) 


\section{Résultats}

Le gain moyen quotidien de poids vif des porcs, au cours de la période comprise entre le jour de l'opération et l'abattage (soit au total 35 jours) s'avère analogue pour une même source protéique, quelle que soit la nature de l'amidon ingéré. Ainsi, le gain de poids moyen quotidien des 6 porcs ayant ingéré un aliment comportant de la farine de poisson est de $597 \pm 39 \mathrm{~g}$ contre seulement $195 \pm 24 \mathrm{~g}$ pour les 6 porcs ayant ingéré un aliment contenant du gluten de blé.

\section{a) Régimes azotés}

\section{1. - Quantités résiduelles dans l'estomac}

Les quantités résiduelles de matière frầche et de matière sèche collectées en moyenne aux 5 temps après le repas sont indiquées dans la figure 1, séparément pour les régimes à base d'amidon de maîs et ceux à base d'amidon de blé. Il apparaît pour les régimes maïs, et à tous les temps de la cinétique, que l'évacuation de la matière fraîche est beaucoup plus rapide lors d'association au gluten que lors d'association au poisson. Par contre, pour les régimes à base de blé, ce n'est que $7 \mathrm{~h}$ après le repas qu'une véritable différence, de sens inverse, est constatée (évacuation plus rapide de la matière fraîche pour l'aliment blé-poisson que pour l'aliment blé-gluten). En ce qui concerne la matière sèche, l'écart observé selon la nature de la protéine associée à un amidon de maïs se trouve limité aux 2 premières heures postprandiales (évacuation plus rapide de la matière sèche du régime maïs-gluten). Dans le cas des régimes blé, la matière sèche s'avère évacuée plus rapidement pour l'association blé-poisson aux quatrième et septième heures postprandiales.

En d'autres termes et sur la base des quantités de matière sèche résiduelle exprimées en p. 100 de la matière sèche ingérée, il apparaît, pour certains temps de la cinétique seulement, des différences selon la protéine associée à un même amidon (fig. 2 A). Il apparaît aussi que selon la source protéique du régime l'influence de la nature de l'amidon est fort variable (fig. $3 \mathrm{~A}$ ).

Les quantités résiduelles d'amidon, collectées en moyenne aux 5 temps après le repas, et exprimées en p. 100 de l'amidon ingéré, sont représentées dans les figures $2 \mathrm{~B}$ et 3 B. Les profils d'évacuation de l'amidon ne semblent pas fondamentalement différents, avec toutefois certaines différences liées à la source protéique selon les temps considérés (fig. $2 \mathrm{~B}$ ) et une légère différence liée à la nature de l'amidon au moins lors d'association au poisson (fig. 3 B). En ce qui concerne l'azote total, et selon le même mode d'expression, on enregistre l'existence de différences liées à la source protéique (fig. $2 \mathrm{C}$ ) et celle de différences liées à la nature de l'amidon, plus ou moins importantes selon la source protéique associée (fig. $3 \mathrm{C}$ ).

Des descriptions qui précèdent, pour chacune des variables contrôlées, il ressort que les facteurs pris en compte dans l'expérience ne sont pas responsables d'effets simples et faciles à caractériser. Dans la plupart des situations, l'existence d'une interférence entre les facteurs paraît vraisemblable. 


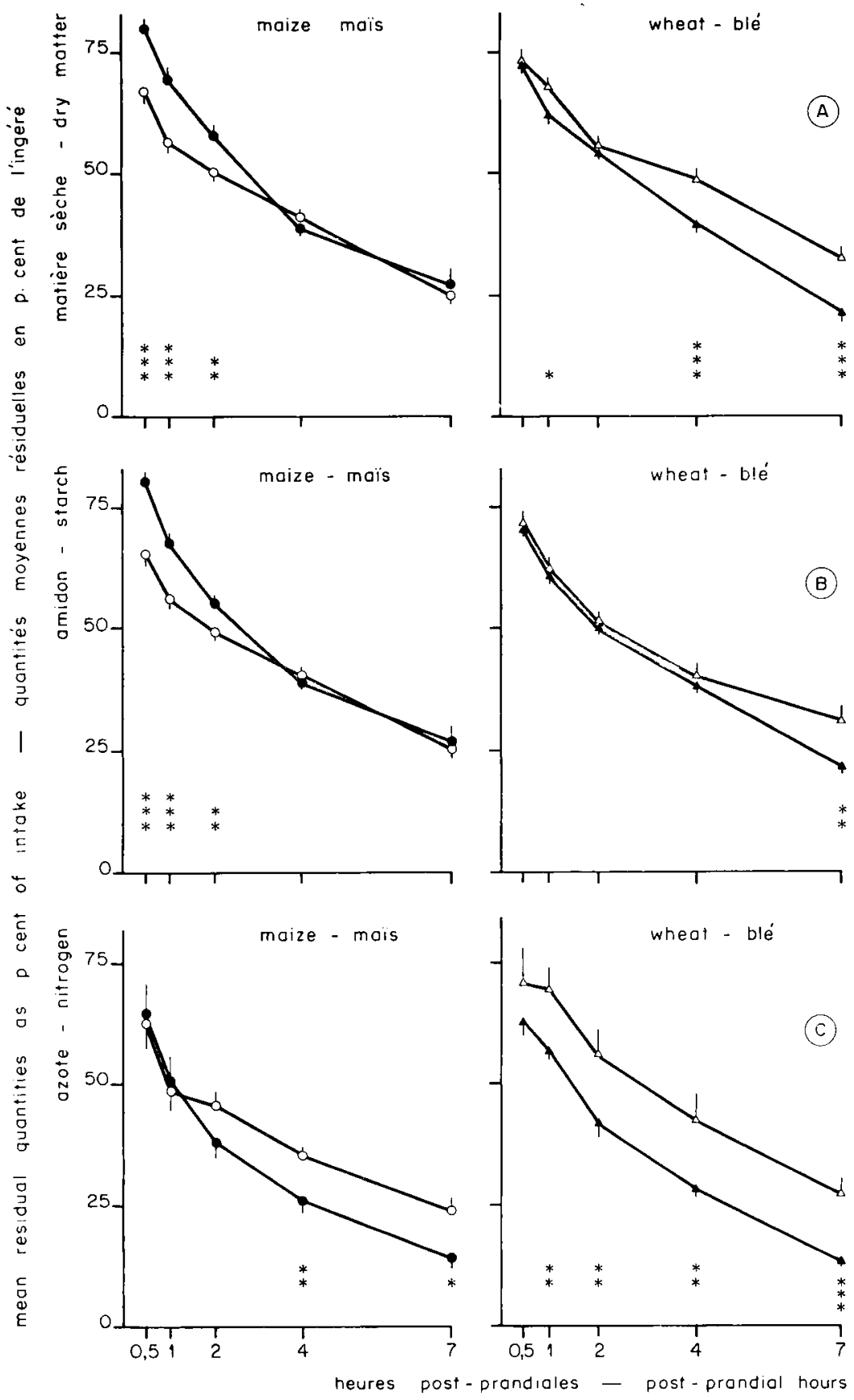

FIG. 2

Quantités moyennes (et écarts types des moyennes) résiduelles dans lestomac, de matière sèche $(A)$, d'amidon $(B)$ et d'azote $(C)$, exprimées en $p .100$ de la quantité ingérée correspondante (voir tableau 1), selon la nature de la source protéique pour un même amidon purifié. Les symboles utilisés sont identiques à ceux de la figure 1.

Mean ( \pm SEM) gastric remnants of dry matter $(A)$, starch $(B)$ and nitrogen $(C)$, expressed as per cent of the corresponding intake (see table 1), according to the protein source for one and the same purified starch. The symbols used are the same as in figure 1. 

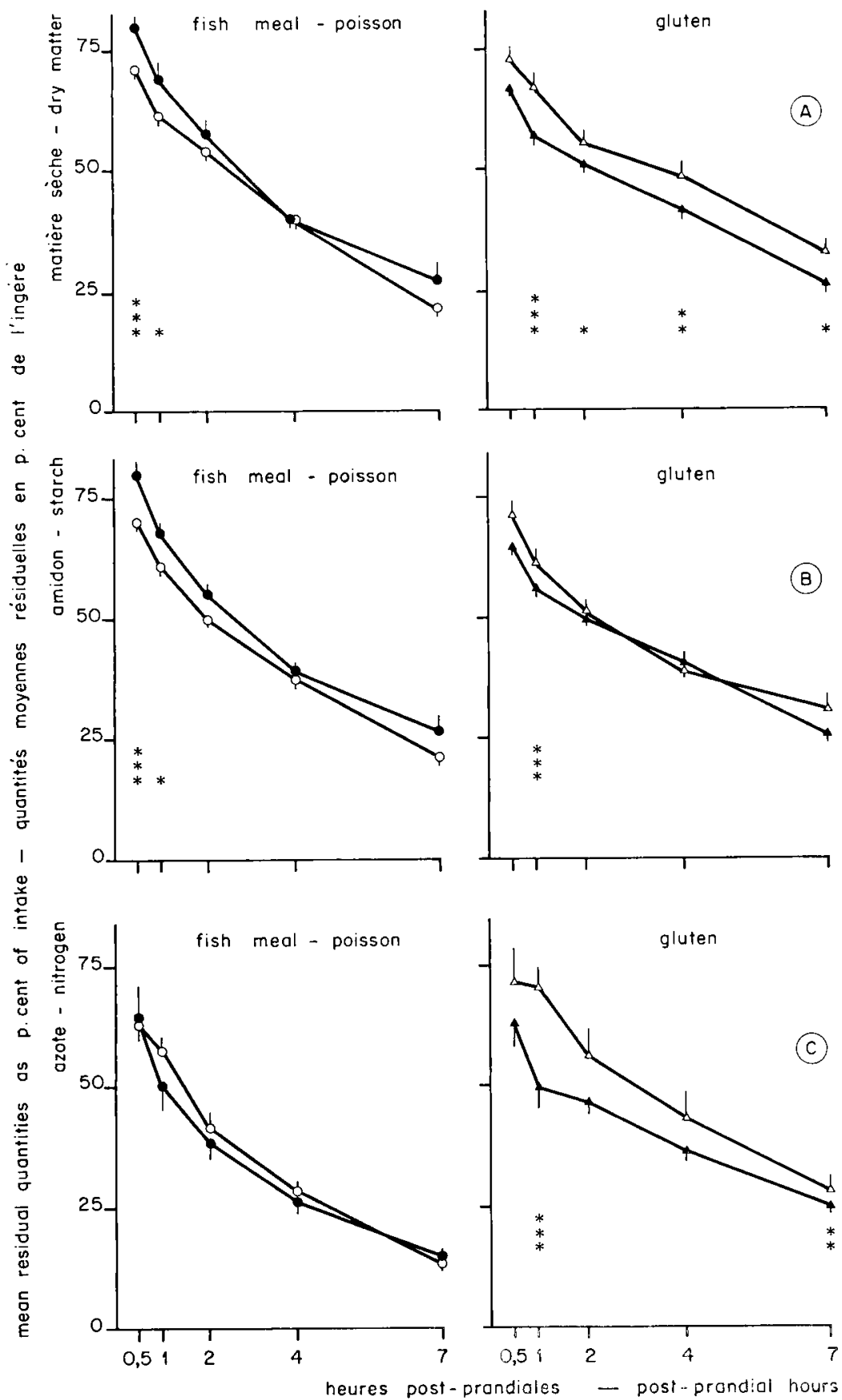

FIG. 3

Quantités moyennes (et écarts types des moyennes) résiduelles dans l'estomac, de matière sèche $(A)$, d'amidon $(B)$ et d'azote $(C)$, exprimées en $p .100$ de la quantité ingérée correspondante (voir tableau 1), selon la nature de l'amidon purifié pour une même source protéique. Astérisques : résultats des tests de Student (voir figure l)

Mean (士 SEM) gastric remnants of dry matter (A), starch (B) and nitrogen (C), expressed as per cent of the corresponding intake (sce table 1), according to the kind of purified starch for one and the same protein source. Asterisks: results of the Student's $t$ test (see figure 1) 


\section{b) Régimes protéiprives}

Les quantités résiduelles de matière fraîche, matière sèche, amidon et azote, recueillies aux temps $2 \mathrm{~h}$ et $7 \mathrm{~h}$ après ingestion d'aliments protéiprives, sont rapportées (en p. 100 des quantités ingérées correspondantes) dans le tableau 2, séparément pour chaque type d'amidon et comparativement aux régimes azotés homologues (base amidon).

Pour une même variable contrôlée et au même temps postprandial, les quantités résiduelles collectées pour l'un ou l'autre des régimes protéiprives (amidon de maïs vs. amidon de blé) ne diffèrent jamais significativement. Les quantités de matière fraîche recueillies respectivement pour les régimes protéiprives et les régimes azotés (moyennes pour un même amidon) ne diffèrent pas sensiblement. Il existe cependant une différence pour les régimes à base d'amidon de maïs entre protéiprive et aliment contenant de la farine de poisson. Au temps $2 \mathrm{~h}$, les quantités résiduelles de matière sèche, amidon et azote, sont toujours plus faibles pour les régimes protéiprives que pour les régimes azotés (moyennes pour un même amidon). Toutefois, une telle différence n'est pas vérifiée en ce qui concerne la matière sèche pour l'association maïsgluten. Au temps $7 \mathrm{~h}$, la quantité résiduelle d'amidon est plus faible pour les régimes protéiprives que pour les régimes azotés (moyennes pour un même amidon). Toutefois, là encore, cette différence n'est pas vérifiée pour l'association blé-poisson.

Le regroupement des données par taux et/ou nature des protéines alimentaires, abstraction faite du type d'amidon servant de base au régime (tableau 3), tend à montrer que la quantité de matière fraîche recueillie $2 \mathrm{~h}$ après les repas protéiprives est analogue à celle collectée pour les régimes gluten, mais significativement plus faible que celle recueillie après les repas d'aliments contenant du poisson. Cette proposition ne peut pourtant être généralisée face à la similitude des valeurs enregistrées pour les régimes à base d'amidon de blé (poisson vs. protéiprive) au tableau 2. Ce seul exemple, conduisant à des conclusions différentes selon le regroupement des données, suggère à nouveau l'existence d'une interférence entre les influences respectives de la nature de l'amidon et de la source protéique.

Dans le cas particulier des régimes protéiprives, la description des quantités résiduelles d'azote en pourcentages des quantités ingérées, telle que réalisée jusqu'ici, masque quelque peu la réalité des différences par rapport aux régimes azotés (tableau 4). En effet, pour les régimes azotés la quantité résiduelle d'azote au temps $7 \mathrm{~h}$ est toujours 2 à 3 fois plus faible que celle recueillie au temps $2 \mathrm{~h}$, alors que cette quantité n'évolue pratiquement pas avec le temps lors d'ingestion d'un régime protéiprive. Par ailleurs, la quantité d'azote (en grammes) recueillie après un repas protéiprive est 21 à 25 fois plus faible au temps $2 \mathrm{~h}$, et 9 à 16 fois moindre au temps $7 \mathrm{~h}$ que la quantité collectée à la suite d'ingestion de régimes azotés.

Régimes farine de poisson (Fish meal diets)

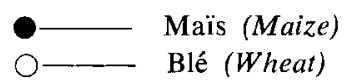

Régimes gluten de blé (Wheat gluten diets)

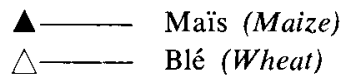


范

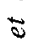

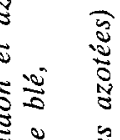

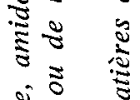
过: यः过 늉ำ 현 ₹ $5 \pm 2$ \& 86 造造 ¿ 0 - $0 气$

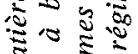

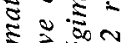
\&:

논

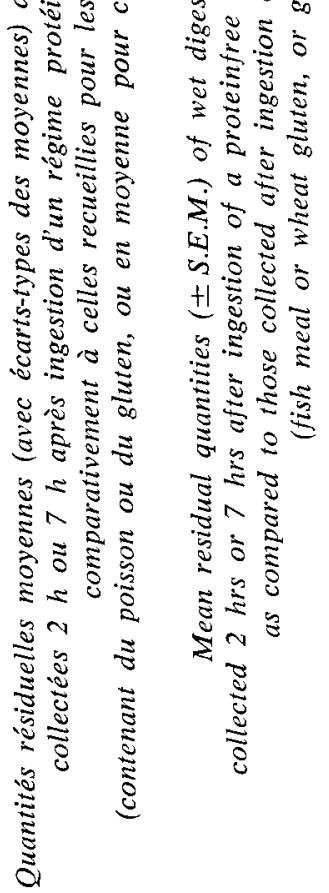

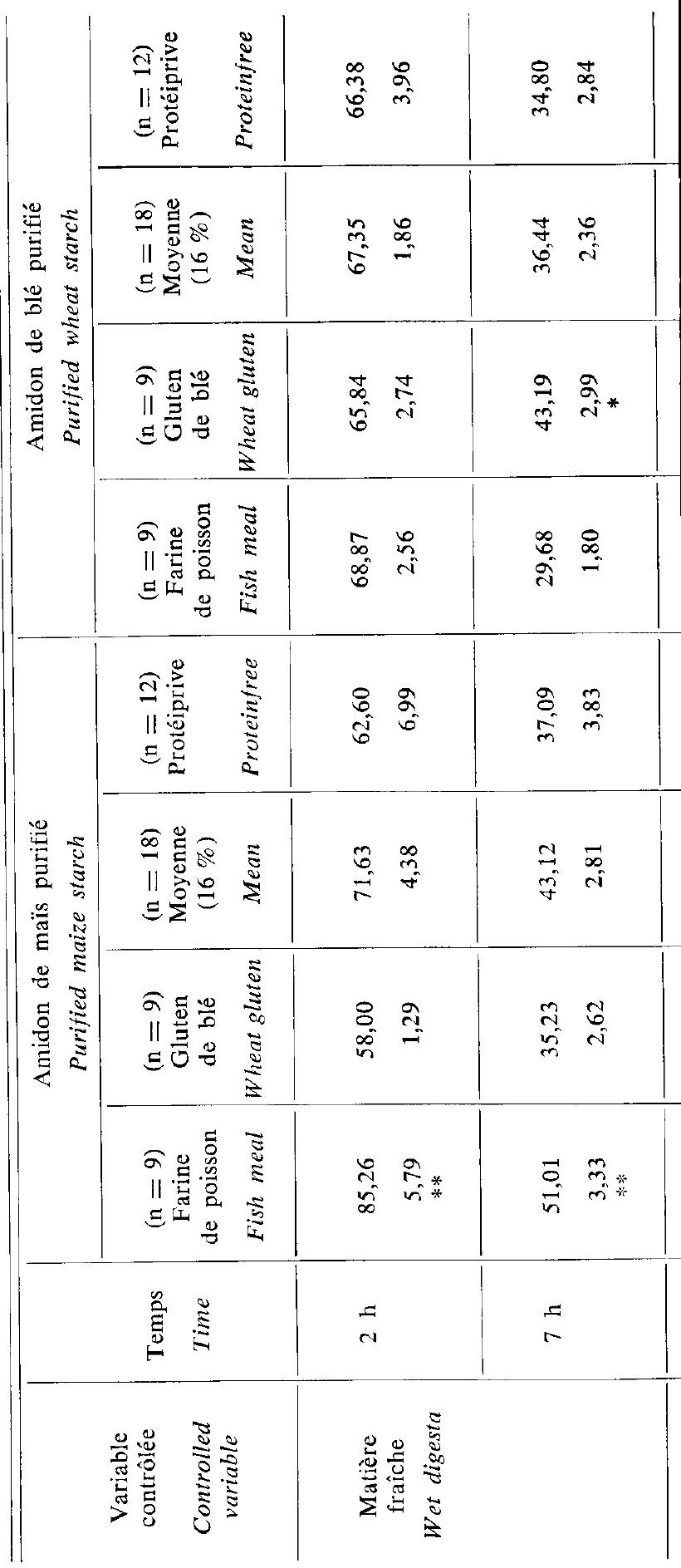




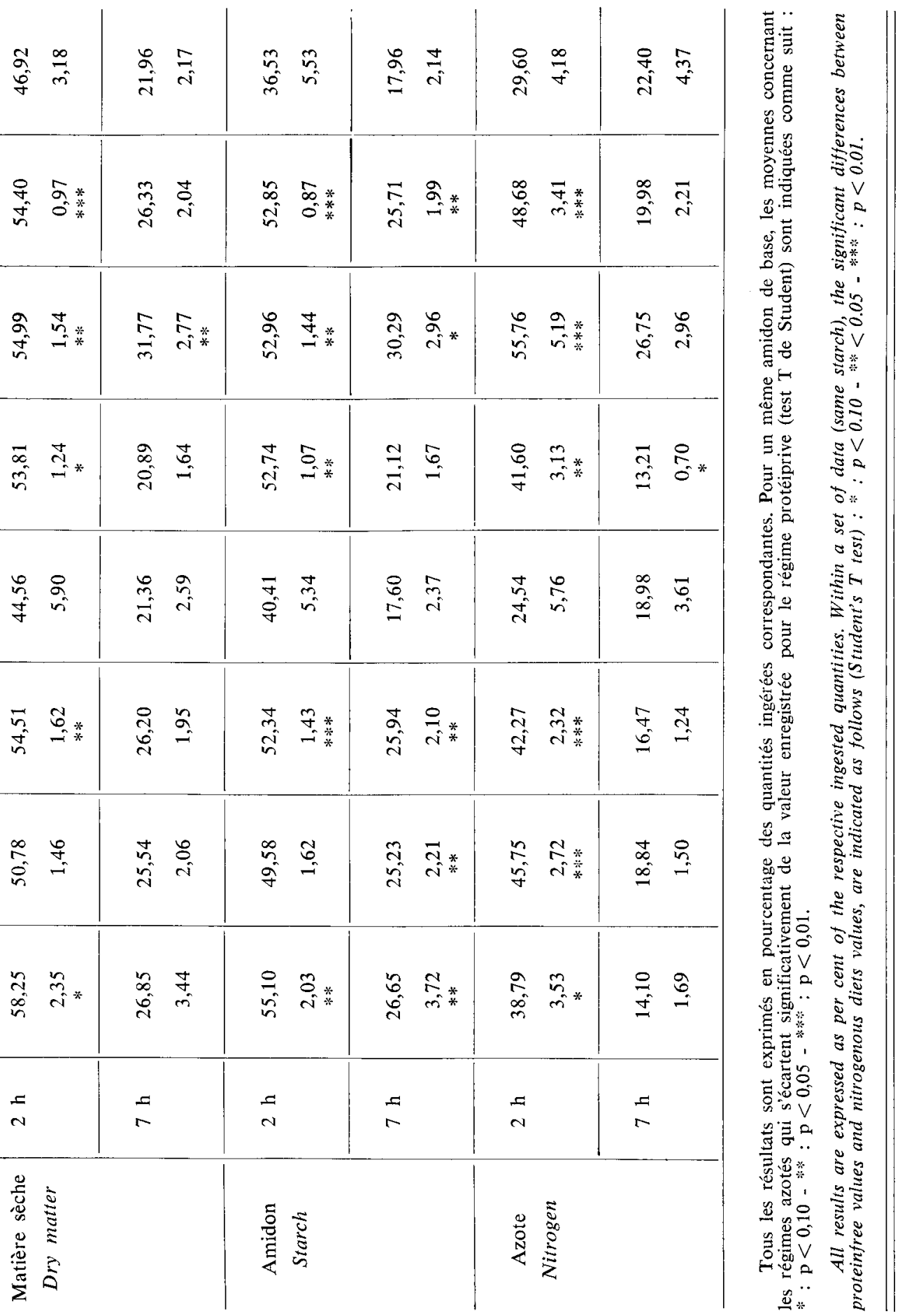




\section{TABLEAU 3}

Quantités résiduelles moyennes (avec écarts-types des moyennes) de matière fraîche, matière sèche, amidon et azote, collectées 2 h ou 7 h après ingestion de régimes protéiprives, comparativement à celles recueillies pour des régimes azotés contenant de la farine de poisson ou du gluten de blé

Pour ces trois cas, les moyennes englobent toutes les mesures, quel que soit l'amidon constituant la base du régime

Mean residual quantities ( \pm S.E.M.) of wet digesta, dry matter, starch and nitrogen, collected $2 \mathrm{hrs}$ or $7 \mathrm{hrs}$ after ingestion of proteinfree diets, as compared to those collected after ingestion of nitrogenous diets including either fish meal or wheat gluten, whatever the kind of starch in all cases

\begin{tabular}{|c|c|c|c|c|}
\hline \multirow{2}{*}{$\begin{array}{c}\text { Variable } \\
\text { contrôlée } \\
\text { Controlled } \\
\text { variable }\end{array}$} & \multirow{2}{*}{$\begin{array}{l}\text { Temps } \\
\text { Time }\end{array}$} & \multicolumn{3}{|c|}{$\begin{array}{c}\text { Deux amidons confondus } \\
\text { Both starches together }\end{array}$} \\
\hline & & $\begin{array}{l}(\mathrm{n}=18) \\
\text { Farine de poisson } \\
\text { Fish meal }\end{array}$ & $\begin{array}{l}\quad(\mathrm{n}=18) \\
\text { Gluten de blé } \\
\text { Wheat gluten }\end{array}$ & $\begin{array}{l}(\mathrm{n}=24) \\
\text { Protéiprive } \\
\text { Proteinfree }\end{array}$ \\
\hline \multirow[t]{2}{*}{$\begin{array}{l}\text { Matière fraîche } \\
\text { Wet digesta }\end{array}$} & $2 \mathrm{~h}$ & $\begin{array}{c}77,06 \\
3,66 \\
* *\end{array}$ & $\begin{array}{r}61,92 \\
1,75\end{array}$ & $\begin{array}{r}64,49 \\
3,94\end{array}$ \\
\hline & $7 \mathrm{~h}$ & $\begin{array}{r}40,34 \\
3,17\end{array}$ & $\begin{array}{r}39,21 \\
2,16\end{array}$ & $\begin{array}{r}35,94 \\
2,34\end{array}$ \\
\hline \multirow[t]{2}{*}{$\begin{array}{l}\text { Matière sèche } \\
\text { Dry matter }\end{array}$} & $2 \mathrm{~h}$ & $\begin{array}{c}56,03 \\
1,40 \\
* *\end{array}$ & $\begin{array}{c}52,88 \\
1,15 \\
*\end{array}$ & $\begin{array}{r}45,74 \\
3,28\end{array}$ \\
\hline & $7 \mathrm{~h}$ & $\begin{array}{r}23,26 \\
2,08\end{array}$ & $\begin{array}{c}28,65 \\
1,8 ? \\
* * *\end{array}$ & $\begin{array}{r}21,67 \\
1,66\end{array}$ \\
\hline \multirow[t]{2}{*}{$\begin{array}{l}\text { Amidon } \\
\text { Starch }\end{array}$} & $2 \mathrm{~h}$ & $\begin{array}{c}53,92 \\
1,15 \\
* * *\end{array}$ & $\begin{array}{c}51,27 \\
1,13 \\
* * k * *\end{array}$ & $\begin{array}{r}41,74 \\
2,96\end{array}$ \\
\hline & $7 \mathrm{~h}$ & $\begin{array}{c}23,88 \\
2,09 \\
* *\end{array}$ & $\begin{array}{c}27,76 \\
1,89 \\
* * *\end{array}$ & $\begin{array}{r}17,78 \\
1,56\end{array}$ \\
\hline \multirow[t]{2}{*}{$\begin{array}{c}\text { Azote } \\
\text { Nitrogen }\end{array}$} & $2 \mathrm{~h}$ & $\begin{array}{c}40,20 \\
2,32 \\
* *\end{array}$ & $\begin{array}{c}50,75 \\
3,09 \\
* * *\end{array}$ & $\begin{array}{r}27,07 \\
3,52\end{array}$ \\
\hline & $7 \mathrm{~h}$ & $\begin{array}{c}13,65 \\
0,89 \\
* *\end{array}$ & $\begin{array}{r}22,79 \\
1,87\end{array}$ & $\begin{array}{r}20,69 \\
2,79\end{array}$ \\
\hline
\end{tabular}

Tous les résultats sont exprimés en pourcentage des quantités ingérées correspondantes. La signification de l'écart de l'un ou l'autre des régimes azotés par rapport aux régimes protéiprives est exprimée (test $t$ de Student) comme suit : * : p<0,10-**: $\mathrm{p}<0,05-* * *: \mathrm{p}<0,01$.

All results are expressed as per cent of the respective ingested quantities. Significant differences between either fish meal or wheat gluten values as compared to proteinfree values, are indicated as follows (Student's $T$ test) : *:p<0.10-**:p<0.05-****:p<0.01. 


\section{Tableau 4}

Quantités résiduelles moyennes (avec écarts-types des moyennes) d'azote, exprimées en grammes, collectées $2 h$ ou $7 h$ après ingestion de régimes protéiprives à base d'amidon de maïs ou de blé, comparativement à celles recueillies pour les régimes azotés correspondants (contenant du poisson ou du gluten)

Mean residual quantities ( \pm S.E.M.) of nitrogen, expressed as grams, collected 2 hrs or 7 hrs after ingestion of a proteinfree diet based on either maize or wheat starch, as compared to those collected after ingestion of the respective nitrogenous diets (fish meal or wheat gluten)

\begin{tabular}{|c|c|c|c|}
\hline \multirow{2}{*}{$\begin{array}{l}\text { Amidon purifié } \\
\text { Purified starch }\end{array}$} & \multirow{2}{*}{$\begin{array}{l}\text { Source de protéines } \\
\text { Protein source }\end{array}$} & \multicolumn{2}{|c|}{ Temps (Time) } \\
\hline & & $2 \mathrm{~h}$ & $7 \mathrm{~h}$ \\
\hline \multirow[t]{3}{*}{$\begin{array}{l}\text { Amidon de maïs } \\
\text { Maize starch }\end{array}$} & $\begin{array}{l}\text { Farine de poisson } \\
\text { Fish meal }\end{array}$ & $9,23 \pm 0,84$ & $3,36 \pm 0,40$ \\
\hline & $\begin{array}{l}\text { Gluten de blé } \ldots \ldots \ldots \ldots \ldots \\
\text { Wheat gluten }\end{array}$ & $10,52 \pm 0,63$ & $4,33 \pm 0,34$ \\
\hline & $\begin{array}{l}\text { Protéiprive } \ldots \ldots \ldots \ldots \ldots \ldots \\
\text { Protein free }\end{array}$ & $0,44 \pm 0,10$ & $0,34 \pm 0,06$ \\
\hline \multirow[t]{3}{*}{$\begin{array}{l}\text { Amidon de blé } \\
\text { Wheat starch }\end{array}$} & $\begin{array}{l}\text { Farine de poisson } \\
\text { Fish meal }\end{array}$ & $9,90 \pm 0,75$ & $3,14 \pm 0,17$ \\
\hline & $\begin{array}{l}\text { Gluten de blé } \ldots \ldots \ldots \ldots \ldots \\
\text { Wheat gluten }\end{array}$ & $12,04 \pm 1,12$ & $5,78 \pm 0,64$ \\
\hline & $\begin{array}{l}\text { Protéiprive .. } \\
\text { Proteinfree }\end{array}$ & $0,47 \pm 0,07$ & $0,36 \pm 0,07$ \\
\hline
\end{tabular}

\section{a) Régimes azotés}

\section{2. - Analyses de variance}

L'ensemble des résultats fournis par les diverses analyses de variance testant les effets amidon, protéine, porc, et leurs interactions, sur les quantités évacuées par l'estomac après ingestion des régimes azotés, est réuni sous forme de tableaux.

Selon les analyses réalisées séparément sur les groupes de données obtenues à chacun des instants d'observation (tableau 5), on note les faits suivants :

- L'effet amidon ne se manifeste guère qu'exceptionnellement, à l'un ou l'autre des temps de collecte, à l'égard des quantités évacuées de matière fraîche, matière sèche et amidon; il est par contre presque constamment enregistré pour ce qui concerne l'azote.

- L'influence de la source protéique s'avère très fréquemment significative à l'égard des quatre variables étudiées : quantités évacuées de matière fraîche, matière sèche, amidon et azote. 


\section{TABleau 5}

Effets expérimentaux significatifs enregistrés lors d'ingestion des régimes azotés selon les analyses de variance réalisées séparément sur ( 3 porcs $\times 3$ collectes $\times 4$ régimes) soit sur 36 données pour chaque instant d'observation et chaque variable étudiée

Significant experimental effects recorded in the case of ingestion of the nitrogenous diets, according to the variance analyses separately performed on ( 3 pigs $\times 3$ collections $\times 4$ diets $)$ i.e. on 36 data for each collection time and each controlled variable

\begin{tabular}{|c|c|c|c|c|c|c|c|c|}
\hline \multicolumn{2}{|c|}{$\begin{array}{l}\text { Effets } \\
\text { Effects }\end{array}$} & \multicolumn{3}{|c|}{$\begin{array}{l}\text { Indépendants } \\
\text { Independent }\end{array}$} & \multicolumn{4}{|c|}{$\begin{array}{l}\text { Interactions } \\
\text { Interactions }\end{array}$} \\
\hline $\begin{array}{l}\text { Variable } \\
\text { Variable }\end{array}$ & $\begin{array}{l}\text { Temps } \\
\text { Time }\end{array}$ & $a$ & b & $\mathrm{c}$ & $a b$ & $\mathrm{ac}$ & bc & $a b c$ \\
\hline $\begin{array}{c}\text { Matière } \\
\text { fraîche } \\
\text { Wet } \\
\text { digesta }\end{array}$ & $\mid \begin{array}{rl}30 & \mathrm{mn} \\
1 & \mathrm{~h} \\
2 & \mathrm{~h} \\
4 & \mathrm{~h} \\
7 & \mathrm{~h}\end{array}$ & $\begin{array}{l}0,195 \\
0,031 \\
2,578 \\
0,490 \\
9,094^{*}\end{array}$ & $\begin{array}{c}41,003^{*} \\
21,063^{*} \\
32,266^{*} \\
0,631 \\
0,261\end{array}$ & $\begin{array}{l}1,007 \\
0,238 \\
1,442 \\
2,308 \\
3,416^{*}\end{array}$ & $\begin{array}{r}7,390^{*} \\
16,077^{*} \\
20,641^{*} \\
4,306^{*} \\
43,719^{*}\end{array}$ & $\begin{array}{l}3,232 \\
4,081^{*} \\
9,068^{*} \\
3,156 \\
6,882^{*}\end{array}$ & $\begin{array}{l}0,702 \\
0,148 \\
4,731 * \\
0,751 \\
1,500\end{array}$ & $\begin{array}{l}3,592 * \\
1,024 \\
0,451 \\
0,102 \\
0,739\end{array}$ \\
\hline $\begin{array}{l}\text { Matière } \\
\text { sèche } \\
\text { Dry } \\
\text { matter }\end{array}$ & $\begin{array}{rl}30 & \mathrm{mn} \\
1 & \mathrm{~h} \\
2 & \mathrm{~h} \\
4 & \mathrm{~h} \\
7 & \mathrm{~h}\end{array}$ & $\begin{array}{c}0,505 \\
1,553 \\
0,006 \\
16,370^{*} \\
0,005\end{array}$ & $\begin{array}{c}15,638^{*} \\
3,861 \\
4,290^{*} \\
34,789^{*} \\
6,052^{*}\end{array}$ & $\begin{array}{l}1,306 \\
1,741 \\
0,269 \\
9,227^{*} \\
8,325^{*}\end{array}$ & $\begin{array}{r}17,387^{*} \\
24,503^{*} \\
7,784^{*} \\
14,413^{*} \\
9,836^{*}\end{array}$ & $\begin{array}{c}5,696^{*} \\
2,773 \\
1,289 \\
17,651 * \\
0,273\end{array}$ & $\begin{array}{l}2,912 \\
2,498 \\
2,323 \\
1,401 \\
1,802\end{array}$ & $\begin{array}{l}1,553 \\
3,934^{*} \\
1,358 \\
7,259^{*} \\
5,636^{*}\end{array}$ \\
\hline $\begin{array}{l}\text { Amidon } \\
\text { Starch }\end{array}$ & $\begin{array}{rl}30 & \mathrm{mn} \\
1 & \mathrm{~h} \\
2 & \mathrm{~h} \\
4 & \mathrm{~h} \\
7 & \mathrm{~h}\end{array}$ & $\begin{array}{c}0,270 \\
3,395 \\
3,008 \\
31,751^{*} \\
2,265\end{array}$ & $\begin{array}{r}22,249 * \\
8,104 * \\
15,381 * \\
0,047 \\
0,386\end{array}$ & $\begin{array}{l}1,192 \\
1,026 \\
0,606 \\
2,262 \\
6,099 *\end{array}$ & $\begin{array}{r}17,091^{*} \\
15,653^{*} \\
3,801^{*} \\
12,611^{*} \\
6,063^{*}\end{array}$ & $\begin{array}{c}3,009 \\
1,445 \\
0,261 \\
17,411^{*} \\
0,403\end{array}$ & $\begin{array}{l}1,118 \\
1,364 \\
3,804^{*} \\
1,464 \\
2,714\end{array}$ & $\begin{array}{l}0,077 \\
3,716^{*} \\
0,645 \\
7,939^{*} \\
6,042^{*}\end{array}$ \\
\hline $\begin{array}{c}\text { Azote } \\
\text { Nitrogen }\end{array}$ & $\begin{array}{rl}30 & \mathrm{mn} \\
1 & \mathrm{~h} \\
2 & \mathrm{~h} \\
4 & \mathrm{~h} \\
7 & \mathrm{~h}\end{array}$ & $\begin{array}{r}1,670 \\
24,071^{*} \\
7,569^{*} \\
23,314^{*} \\
14,462^{*}\end{array}$ & $\begin{array}{r}2,860 \\
5,698^{*} \\
24,321^{*} \\
157,038^{*} \\
91,150^{*}\end{array}$ & $\begin{array}{r}5,309^{*} \\
7,676^{*} \\
4,035^{*} \\
16,363^{*} \\
3,879^{*}\end{array}$ & $\begin{array}{c}2,767 \\
6,307^{*} \\
2,990 \\
11,059^{*} \\
19,471^{*}\end{array}$ & $\begin{array}{r}3,516^{*} \\
5,579^{*} \\
6,151^{*} \\
19,015^{*} \\
4,047^{*}\end{array}$ & $\begin{array}{l}5,309^{*} \\
3,434^{*} \\
0,817 \\
2,368 \\
0,397\end{array}$ & $\begin{array}{c}17,976^{*} \\
1,795 \\
4,401 * \\
35,969^{*} \\
3,581^{*}\end{array}$ \\
\hline
\end{tabular}

Les valeurs numériques sont celles fournies par le test $F$ de Fisher; les effets significatifs (au seuil 5 p. 100) selon ces valeurs sont indiqués par un astérisque.

$a=$ effet amidon; $b=$ effet protéine; $c=$ effet porc; $a b, a c, b c, a b c=$ interactions entre les effets correspondants.

The values given were provided by the Fisher's $F$ test; the significant effects $(5$ p. 100$)$ are indicated by asterisks.

$a=$ starch effect $; b=$ protein effect ; $c=$ pig effect $; a b, a c, b c, a b c=$ interactions between the respective effects. 


\section{TABLeau 6}

Effets expérimentaux significatifs enregistrés lors d'ingestion des régimes azotés selon l'analyse de variance multidimensionnelle portant sur (3 porcs $\times 3$ collectes $\times 5$ temps $\times 4$ régimes $)$ soit sur 180 données pour chaque variable étudiée

Significant experimental effects recorded in the case of ingestion of the nitrogenous diets according to the overall result of the multivariate analysis of variance performed on ( 3 pigs $\times 3$ collections $\times 5$ times $\times 4$ diets $)$ i.e. on 180 data for each controlled variable

\begin{tabular}{|c|c|c|c|c|c|c|c|}
\hline \multirow{2}{*}{$\begin{array}{c}\text { Effets } \\
\text { Effects } \\
\text { Variable étudiée } \\
\text { Studied variable }\end{array}$} & \multicolumn{3}{|c|}{$\begin{array}{l}\text { Indépendants } \\
\text { Independent }\end{array}$} & \multicolumn{4}{|c|}{$\begin{array}{l}\text { Interactions } \\
\text { Interactions }\end{array}$} \\
\hline & $\mathrm{a}$ & b & $\mathrm{c}$ & $a b$ & $\mathrm{ac}$ & $b c$ & $a b c$ \\
\hline $\begin{array}{l}\text { Matière fraîche } \ldots \ldots \\
\text { Wet digesta }\end{array}$ & 0,3172 & $0,7758^{*}$ & 0,3572 & $0,7794 *$ & $0,6927^{*}$ & 0,4036 & 0,2988 \\
\hline $\begin{array}{l}\text { Matière sèche } \ldots \\
\text { Dry matter }\end{array}$ & $0,4971^{*}$ & $0,6987^{*}$ & 0,5448 & $0,6979 *$ & $0,7187^{*}$ & 0,3442 & 0,4391 \\
\hline $\begin{array}{l}\text { Amidon } \\
\text { Starch }\end{array}$ & $0,6327^{*}$ & $0,6200^{*}$ & 0,4460 & $0,6839 *$ & $0,6847^{*}$ & 0,3259 & $0,4539^{*}$ \\
\hline $\begin{array}{l}\text { Azote } \ldots \ldots \ldots \ldots \\
\text { Nitrogen }\end{array}$ & $0,6946^{*}$ & $0,9421^{*}$ & $0,7030^{*}$ & $0,6030^{*}$ & $0,7674^{*}$ & $0,5351 *$ & $0,8426^{*}$ \\
\hline
\end{tabular}

Les valeurs numériques sont celles du test de Roy; les effets significatifs (au seuil 5 p. 100) selon ces valeurs sont indiqués par un astérisque.

$\mathrm{a}=$ effet amidon $; \mathrm{b}=$ effet protéine $; \mathrm{c}=$ effet porc $; \mathrm{ab}, \mathrm{ac}, \mathrm{bc}, \mathrm{abc}=$ interactions entre les effets correspondants.

The values given were provided by the Roy's test; the significant effects $(5 \mathrm{p} .100)$ are indicated by asterisks.

$a=$ starch effect $; b=$ protein effect $; c=$ pig effect $; a b, a c, b c, a b c=$ interactions between the respective effects.

- L'effet porc est significatif pour un certain nombre de collectes à l'égard des quantités évacuées de matière fraîche, matière sèche et amidon, notamment au temps $7 \mathrm{~h}$ après le repas; il est constamment présent pour ce qui concerne les quantités d'azote évacuées par l'estomac.

- L'interaction entre l'amidon et la protéine du régime à l'égard de l'évacuation de l'estomac apparaît comme l'effet dominant, enregistré presque constamment pour les quatre variables étudiées.

- Les interactions amidon-porc et protéine-porc sont relativement fréquemment significatives, en particulier à l'égard des quantités d'azote évacuées par l'estomac. 
- L'interaction amidon-protéine-porc, significative à certains instants d'observation pour la matière fraîche, la matière sèche et l'amidon, est encore presque constamment significative pour ce qui concerne l'azote.

L'analyse de variance multidimensionnelle testant globalement sur l'ensemble des observations expérimentales les mêmes effets (tableau 6), fournit une vue plus synthétique des influences significatives. Les trois tests de RoY, de WILKs et de LaWLeYHotTEling fournissant des résultats parfaitement concordants, seuls sont rapportés ceux du test de Roy. Il apparaît que :

- L'évacuation gastrique de la matière sèche, de l'amidon et de l'azote, est significativement affectée par la nature de l'amidon du régime.

- L'influence de la source de protéines alimentaires s'exerce sur toutes les variables contrôlées.

- L'effet de l'animal est significatif pour ce qui concerne l'évacuation gastrique de la matière sèche et de l'azote.

- Les interactions entre la nature de l'amidon et la source de protéines d'une part, entre la nature de l'amidon et l'animal d'expérience d'autre part sont significatives pour toutes les variables contrôlées.

- L'interaction entre la source de protéines et le porc est enregistrée seulement à l'égard de l'évacuation de l'azote.

- Enfin, l'interaction amidon-protéine-porc est constatée dans le cas de l'évacuation de l'amidon et de l'azote.

b) Régimes protéiprives

Les résultats enregistrés, dans les analyses de variance réalisées séparément pour chacun des 2 instants d'observation ( $2 \mathrm{~h}$ et $7 \mathrm{~h}$ pour les régimes protéiprives) et dans lanalyse de variance multidimensionnelle, sont totalement cohérents (tableau 7).

On enregistre ainsi :

- Un effet porc et une interaction amidon-porc à l'égard de l'évacuation de la matière fraîche.

- Un effet exclusif de l'animal d'expérience pour ce qui concerne l'évacuation de la matière sèche et de l'amidon.

- Des effets de lamidon d'une part et du porc d'autre part à l'égard de l'évacuation de lazote.

\section{3. - Décomposition polynomiale des effets (régimes azotés)}

Les tests de nullité réalisés sur les coefficients polynomiaux (matrice $\theta_{2}$ ), résultant des ajustements pour chacun des effets et interactions du modèle, sont cohérents quelle que soit la méthode mise en cuvre (Roy, Wilks ou LaWLEY-HotTeling). Il apparaît que le terme de degré 4 (degré maximum pour 5 instants d'observation) est constamment nul. Le terme de degré 3 s'avère nul dans le cas de la matière fraîche, mais significatif pour la matière sèche, l'amidon et l'azote. 


\section{TABLEAU 7}

Effets expérimentaux significatifs enregistrés lors d'ingestion de régimes protéiprives selon les deux types d'analyse de variance

Significant experimental effects recorded in the case of ingestion of the proteinfree diets according to the two kinds of variance analysis performed

\begin{tabular}{|c|c|c|c|c|c|}
\hline \multicolumn{3}{|c|}{$\begin{array}{l}\text { Effets } \\
\text { Effects }\end{array}$} & \multicolumn{2}{|c|}{$\begin{array}{l}\text { Indépendants } \\
\text { Independent }\end{array}$} & \multirow{2}{*}{$\begin{array}{l}\text { Interaction } \\
\text { Interaction } \\
\quad \mathrm{ac}\end{array}$} \\
\hline $\begin{array}{l}\text { Variable étudiée } \\
\text { Studied variable }\end{array}$ & \multicolumn{2}{|c|}{$\begin{array}{l}\text { Type d'analyse de variance } \\
\text { Kind of variance analysis }\end{array}$} & $\begin{array}{l}\text { a } \\
\text { Effet amidon } \\
\text { Starch effect }\end{array}$ & $\begin{array}{l}\mathrm{c} \\
\text { Effet porc } \\
\text { Pig effect }\end{array}$ & \\
\hline \multirow[t]{2}{*}{$\begin{array}{l}\text { Matière fraîche } \\
\text { Wet digesta }\end{array}$} & $\begin{array}{c}\text { Par instant } \\
\text { d'observation } \\
\text { At each collection } \\
\text { time }\end{array}$ & $\begin{array}{l}2 \mathrm{~h} \\
7 \mathrm{~h}\end{array}$ & $\begin{array}{l}0,691 \\
0,220\end{array}$ & $\begin{array}{l}4,975^{*} \\
4,799 *\end{array}$ & $\begin{array}{l}1,221 \\
5,585^{*}\end{array}$ \\
\hline & \multicolumn{2}{|c|}{$\begin{array}{c}\text { Globale, } \\
\text { multidimensionnelle } \\
\text { Overall, multivariate }\end{array}$} & 0,0601 & $0,699 *$ & $0,7042 *$ \\
\hline \multirow[t]{2}{*}{$\begin{array}{l}\text { Matière sèche } \\
\text { Dry matter }\end{array}$} & $\begin{array}{c}\text { Par instant } \\
\text { d'observation } \\
\text { At each collection } \\
\text { time }\end{array}$ & $\begin{array}{l}2 \mathrm{~h} \\
7 \mathrm{~h} \\
\end{array}$ & $\begin{array}{l}1,287 \\
0,060\end{array}$ & $\begin{array}{l}4,837^{*} \\
3,706^{*}\end{array}$ & $\begin{array}{l}0,879 \\
2,427 \\
\end{array}$ \\
\hline & \multicolumn{2}{|c|}{$\begin{array}{l}\text { Globale, } \\
\text { multidimensionnelle } \\
\text { Overall, multivariate }\end{array}$} & 0,0969 & $0,6808^{*}$ & 0,5061 \\
\hline \multirow[t]{2}{*}{$\begin{array}{l}\text { Amidon } \\
\text { Starch }\end{array}$} & $\begin{array}{c}\text { Par instant } \\
\text { d'observation } \\
\text { At each collection } \\
\text { time }\end{array}$ & $\begin{array}{l}2 \mathrm{~h} \\
7 \mathrm{~h}\end{array}$ & $\begin{array}{l}0,346 \\
0,048\end{array}$ & $\begin{array}{l}4,683^{*} \\
5,016^{*} \\
\end{array}$ & $\begin{array}{l}1,116 \\
2,347 \\
\end{array}$ \\
\hline & \multicolumn{2}{|c|}{$\begin{array}{c}\text { Globale, } \\
\text { multidimensionnelle } \\
\text { Overall, multivariate }\end{array}$} & 0,0412 & $0,7145^{*}$ & 0,4945 \\
\hline \multirow[t]{2}{*}{$\begin{array}{c}\text { Azote } \\
\text { Nitrogen }\end{array}$} & $\begin{array}{c}\text { Par instant } \\
\text { d'observation } \\
\text { At each collection } \\
\text { time }\end{array}$ & $\begin{array}{l}2 \mathrm{~h} \\
7 \mathrm{~h}\end{array}$ & $\begin{array}{l}6,568^{*} \\
2,376\end{array}$ & $\begin{array}{l}4,211^{*} \\
0,910\end{array}$ & $\begin{array}{l}1,184 \\
0,734\end{array}$ \\
\hline & \multicolumn{2}{|c|}{$\begin{array}{c}\text { Globale, } \\
\text { multidimensionnelle } \\
\text { Overall, multivariate }\end{array}$} & $0,5009 *$ & $0,6667 *$ & 0,4763 \\
\hline
\end{tabular}

Pour les analyses par instant d'observation, test $\mathrm{F}$ de Fisher ; pour l'analyse multidimensionnelle, test de Roy. Les effets significatifs (au seuil 5 p. 100 dans les 2 cas) sont indiqués par un astérisque.

For the analyses done at each collection time, Fisher's $F$ test; for the multivariate analysis, Roy's test. The significant effects (5 p. 100 in both cases) are indicated by asterisks. 
Régressions polynomiales exprimant pour chacune des variables contrôlées la quantité évacuée $Y$ en fonction du temps après ingestion des régimes azotés

Polynomial regressions representative, for each of the controlled variables, of the emptied quantity $Y$ as a function of the time after intake of the nitrogenous diets

\begin{tabular}{|c|c|c|c|c|c|c|}
\hline \multirow{2}{*}{$\begin{array}{l}Y=\text { quantité } \\
\text { évacuée } \\
Y=\text { emptied } \\
\text { quantity }\end{array}$} & \multirow{2}{*}{$\begin{array}{c}\text { Nature } \\
\text { de l'amidon } \\
\text { purifié } \\
\text { Kind of } \\
\text { purified } \\
\text { starch }\end{array}$} & \multirow{2}{*}{$\begin{array}{c}\text { Source } \\
\text { de protéines } \\
\text { Protein } \\
\text { source }\end{array}$} & \multicolumn{4}{|c|}{$\begin{array}{l}\text { Coefficients de la régression polynomiale } \\
\text { Coefficients of the polynomial regression }\end{array}$} \\
\hline & & & $b_{0}$ & $b_{1}$ & $b_{2}$ & $b_{3}$ \\
\hline \multirow{2}{*}{$\begin{array}{c}\text { Matière } \\
\text { fraîche } \\
\text { Wet } \\
\text { digesta }\end{array}$} & $\begin{array}{c}\text { Blé } \\
\text { Wheat }\end{array}$ & $\begin{array}{c}\text { Poisson } \\
\text { Fish meal } \\
\text { Gluten } \\
\text { Wheat gluten }\end{array}$ & $\begin{array}{r}65,3069 \\
266,6133\end{array}$ & $\begin{array}{l}352,1985 \\
264,1797\end{array}$ & $\begin{array}{l}-16,0565 \\
-14,5149\end{array}$ & \\
\hline & $\begin{array}{l}\text { Maïs } \\
\text { Maize }\end{array}$ & $\begin{array}{c}\text { Poisson } \\
\text { Fish meal } \\
\text { Gluten } \\
\text { Wheat gluten }\end{array}$ & $\begin{array}{r}-197,1135 \\
435,2683\end{array}$ & $\begin{array}{l}382,3535 \\
320,3674\end{array}$ & $\begin{array}{l}-25,2495 \\
-21,8458\end{array}$ & \\
\hline \multirow{2}{*}{$\begin{array}{l}\text { Matière } \\
\text { sèche } \\
\text { Dry } \\
\text { matter }\end{array}$} & $\begin{array}{c}\text { Blé } \\
\text { Wheat }\end{array}$ & $\begin{array}{c}\text { Poisson } \\
\text { Fish meal } \\
\text { Gluten } \\
\text { Wheat gluten }\end{array}$ & $\begin{array}{l}174,1184 \\
147,3610\end{array}$ & $\begin{array}{l}168,6487 \\
190,2801\end{array}$ & $\begin{array}{l}-28,0374 \\
-42,0652\end{array}$ & $\begin{array}{l}2,0861 \\
3,4463\end{array}$ \\
\hline & $\begin{array}{l}\text { Maïs } \\
\text { Maize }\end{array}$ & $\begin{array}{c}\text { Poisson } \\
\text { Fish meal } \\
\text { Gluten } \\
\text { Wheat gluten }\end{array}$ & $\begin{array}{r}97,8405 \\
219,8125\end{array}$ & $\begin{array}{l}177,8259 \\
181,1512\end{array}$ & $\begin{array}{l}-20,8802 \\
-39,6342\end{array}$ & $\begin{array}{l}0,9459 \\
3,2354\end{array}$ \\
\hline \multirow{2}{*}{$\begin{array}{l}\text { Amidon } \\
\text { Starch }\end{array}$} & $\begin{array}{c}\text { Blé } \\
\text { Wheat }\end{array}$ & $\begin{array}{c}\text { Poisson } \\
\text { Fish meal } \\
\text { Gluten } \\
\text { Wheat gluten }\end{array}$ & $\begin{array}{l}133,8562 \\
116,4740\end{array}$ & $\begin{array}{l}108,3981 \\
143,7336\end{array}$ & $\begin{array}{l}-17,2061 \\
-31,2833\end{array}$ & $\begin{array}{l}1,2518 \\
2,5185\end{array}$ \\
\hline & $\begin{array}{l}\text { Maïs } \\
\text { Maize }\end{array}$ & $\begin{array}{c}\text { Poisson } \\
\text { Fish meal } \\
\text { Gluten } \\
\text { Wheat gluten }\end{array}$ & $\begin{array}{r}41,1734 \\
183,5860\end{array}$ & $\begin{array}{l}183,4207 \\
127,9177\end{array}$ & $\begin{array}{l}-33,5522 \\
-26,7947\end{array}$ & $\begin{array}{l}2,2884 \\
2,1626\end{array}$ \\
\hline \multirow[t]{2}{*}{$\begin{array}{c}\text { Azote } \\
\text { Nitrogen }\end{array}$} & $\begin{array}{c}\text { Blé } \\
\text { Wheat }\end{array}$ & $\begin{array}{c}\text { Poisson } \\
\text { Fish meal } \\
\text { Gluten } \\
\text { Wheat gluten }\end{array}$ & $\begin{array}{l}6,1480 \\
4,3800\end{array}$ & $\begin{array}{l}5,1272 \\
2,9577\end{array}$ & $\begin{array}{l}-\quad 0,8002 \\
-\quad 0,2897\end{array}$ & $\begin{array}{l}0,0520 \\
0,0144\end{array}$ \\
\hline & $\begin{array}{l}\text { Maïs } \\
\text { Maize }\end{array}$ & $\begin{array}{c}\text { Poisson } \\
\text { Fish meal } \\
\text { Gluten } \\
\text { Wheat gluten }\end{array}$ & $\begin{array}{l}5,4286 \\
6,6612\end{array}$ & $\begin{array}{l}7,2836 \\
5,2642\end{array}$ & $\begin{array}{l}-\quad 1,5112 \\
-\quad 1,2254\end{array}$ & $\begin{array}{l}0,1110 \\
0,1027\end{array}$ \\
\hline
\end{tabular}

Les termes $b_{0}, b_{1}$, $b_{2}$ et $b_{; 3}$ sont les coefficients des polynômes, de degré 2 ou 3 selon le cas.

The terms $b_{0}, b_{1}, b_{2}$ and $b_{3}$ are the coefficients of the polynoms (degree 2 ou 3 according to the variable). 
Le meilleur ajustement pour tous les termes de lanalyse de variance est donc du degré 2 pour la matière frâiche, et du degré 3 pour matière sèche, amidon et azote, soit de la forme :

$$
\begin{aligned}
& \text { degré } 2: Y=b_{0}+b_{1} t+b_{2} t^{2} \\
& \text { degré } 3: Y=b_{0}+b_{1} t+b_{2} t^{2}+b_{3} t^{3} \\
& \text { avec } Y=\text { quantité évacuée en fonction du temps; } \\
& \qquad \begin{array}{l}
\mathbf{t}=\text { temps. }
\end{array}
\end{aligned}
$$

\section{4. - Comparaison des courbes moyennes (régimes azotés)}

L’ajustement moyen effectué par régression polynomiale sur les données obtenues pour chaque régime ( 3 porcs $\times 3$ cinétiques), sous la contrainte du degré déterminé précédemment pour chaque variable, fournit les polynômes réunis dans le tableau 8 . Notons seulement que le terme $b_{.2}$ est constamment négatif. L'analyse de la signification des valeurs des coefficients de ces polynômes, étroitement dépendante de l'interprétation de ces coefficients, sera envisagée dans la discussion.

Les tests d'égalité et de parallélisme des courbes (représentatives des polynômes) décrivant l'évacuation gastrique en fonction du temps (figure 4) conduisent à enregistrer les points suivants lorsque sont globalement comparées pour une même variable les 4 courbes correspondant aux 4 régimes azotés :

- Le parallélisme d'ensemble des profils d'évacuation gastrique pour les 4 régimes est rejeté dans le cas de la matière fraîche, de la matière sèche et de l'amidon.

- Le parallélisme des 4 profils d'évacuation de l'azote, après ingestion de l'un ou l'autre des 4 régimes, est par contre accepté.

- Il n'y a pas égalité générale des profils d'évacuation enregistrés pour les 4 régimes, quelle que soit la variable étudiée.

Ces mêmes tests d'égalité et de parallélisme, réalisés pour chacune des 6 comparaisons des courbes prises deux à deux fournissent les résultats suivants :

- Pour la matière fraîche, aucune des 4 courbes représentatives des divers régimes n'est égale à une autre, par contre le parallélisme est accepté pour 4 des 6 comparaisons (figure $4 \mathrm{~A}$ ).

- Pour la matière sèche, aucune des 4 courbes n'est égale à une autre, mais il y a parallélisme des 2 courbes correspondant aux régimes comportant de la farine de poisson d'une part, et parallélisme des 2 courbes correspondant aux régimes comportant du gluten de blé d'autre part (figure $4 \mathrm{~B}$ ).

- Pour l'amidon, trois des courbes sont parallèles entre elles, le parallélisme n'étant rejeté, quelle que soit la comparaison, que par rapport à la courbe représentative du régime maïs-poisson. Il y a de surcroît égalité des 2 courbes correspondant aux deux régimes à base d'amidon de blé purifié (figure $4 \mathrm{C}$ ).

- Pour l'azote, trois des courbes peuvent être considérées comme égales, la quatrième (correspondant à l'association amidon de blé-gluten de blé) n'étant que parallèle aux trois autres (figure $4 \mathrm{D}$ ). 

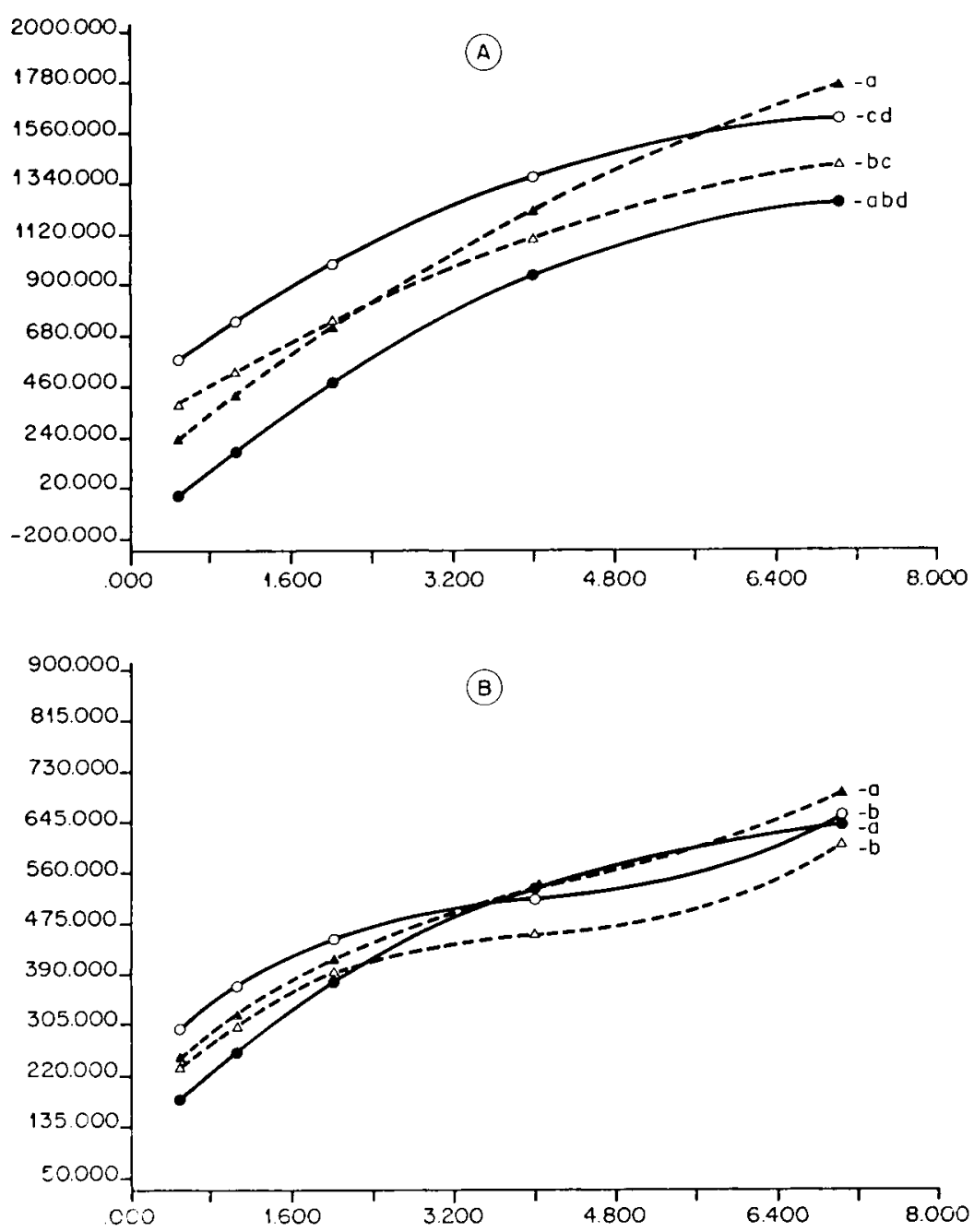

Fig. 4

Courbes, consiruites par lordinateur, décrivant les régressions polynomiales qui expriment

la quantité évacuée $Y$ (en grammes) en fonction du temps $x$ (en heures), pour chacun des 4 régimes azotés et chacune des variables contrôlées : matière fraîche $(A)$; matière sèche $(B)$; amidon $(C)$; azote $(D)$. Des courbes affectées d'une même lettre minuscule peuvent être considérées comme parallèles; des courbes affectées de la lettre $E$ peuvent être considérées comme égales

Régimes maïs (Maize diets)

- Farine de poisson (Fish meal)

O_- Gluten de blé (Wheat gluten)

Régimes blé (Wheat diets)

A..... Farine de poisson (Fish meal)

$\triangle \ldots . .-$ Gluten de blé (Wheat gluten) 

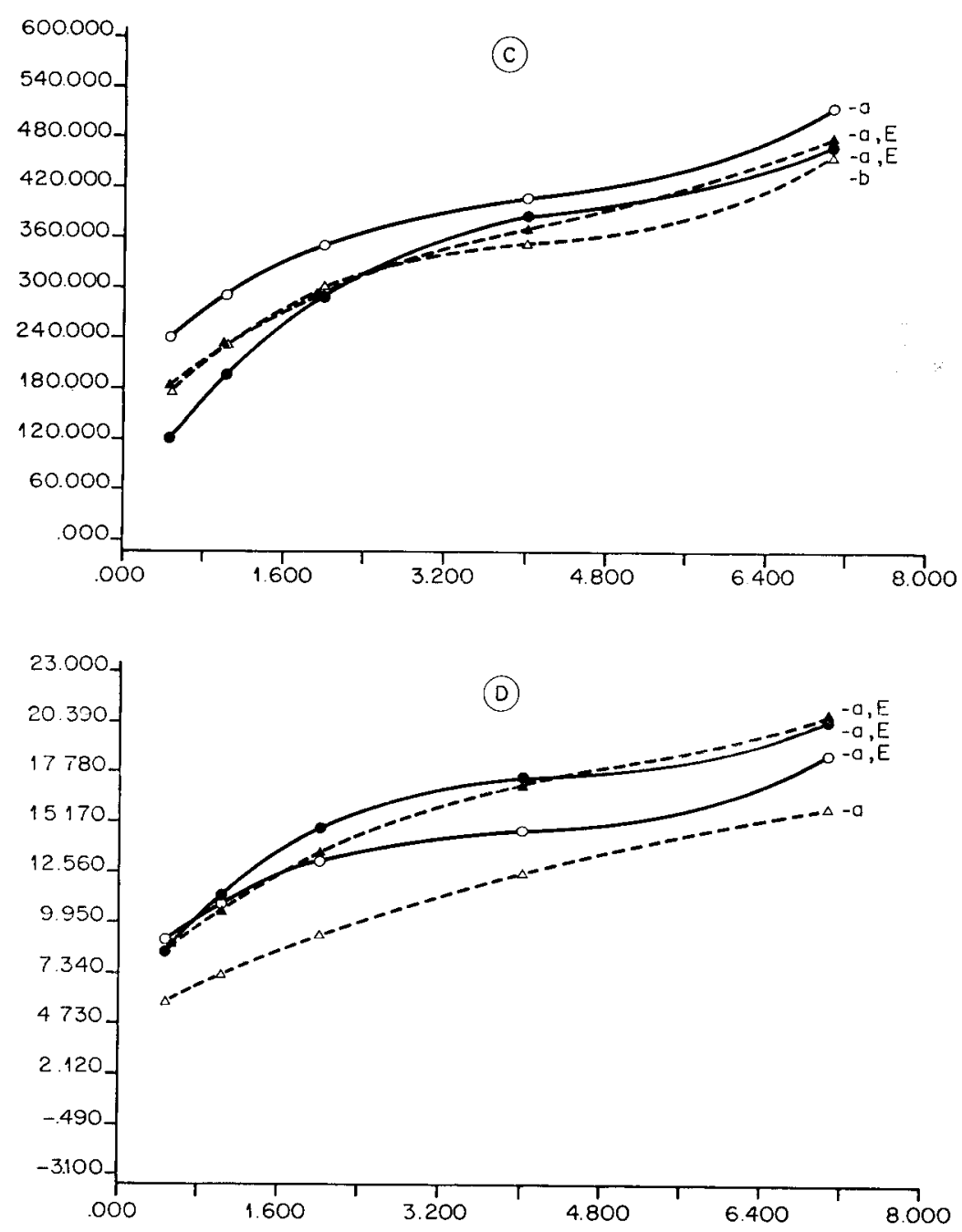

FIG. 4 (suite)

Computer drawn curves based on polynomial regressions representative of the emptied quantity $Y$ (grams) as a function of the time $x$ (hours), for each of the 4 nitrogenous diets and each of the controlled variables : wet digesta $(A) ;$ dry matter $(B) ;$ starch $(C)$; nitrogen (D). Curves indiced by the same small letter may be considered as parallel; curves indiced by the letter $E$ may be considered as equal 


\section{Discussion}

\section{A. - Etude des effets expérimentaux dans le cas des régimes azotés}

\section{1) Les effets indépendants selon l'analyse de variance multidimensionnelle}

Les résultats fournis globalement par l'analyse multidimensionnelle sur l'ensemble des données du schéma factoriel pour les régimes azotés représentent bien la synthèse des effets identifiés par les analyses de variance classiques conduites sur les données afférentes à chaque temps postprandial. C'est donc sur la base des effets et interactions mis en évidence par l'analyse multidimensionnelle que nous dresserons le bilan des effets exercés par lamidon de blé ou de maîs associés à une farine de poisson ou un gluten de blé sur l'évacuation gastrique.

Si l'on considère les effets indépendants, on note l'existence d'une influence de la nature de l'amidon à l'égard de l'évacuation gastrique de la matière sèche, de l'amidon et de l'azote. Cet effet suggère qu'en dépit de la très grande similitude des caractéristiques physico-chimiques de l'amidon de maïs et de l'amidon de blé (GuiLвот, 1968), leur «comportement » dans le tube digestif n'est pas analogue. Toutefois, cette observation reste de pure hypothèse en raison de l'existence d'interactions entre la nature de lamidon et d'autres facteurs expérimentaux.

Un effet de la source de protéines alimentaires est enregistré à l'égard de l'évacuation gastrique de la matière fraîche, de la matière sèche, de lamidon et de l'azote. Les différences répondant à cet effet de la source de protéines pourraient relever de diverses causes telles que une influence de l'appétibilité des régimes, assurément très différente selon qu'ils comportent de la farine de poisson ou du gluten de blé, ou un effet résultant des compositions respectives en acides aminés des deux sources de protéines, le gluten se caractérisant par une faible proportion relative d'acides aminés essentiels dans les acides aminćs totaux et une forte teneur en glutamine et proline par rapport à la farine de poisson (DARCY, 1980). L'écart entre les quantités résiduelles d'azote, selon la source protéique du régime, pourrait aussi être relié à la proportion d'azote soluble dans l'azote ingéré. Celle-ci est beaucoup plus importante dans le cas du poisson (30 p. 100 environ contre 2 p. 100 pour le gluten). Lócart des quantités d'azote total résiduel selon la source protéique ingérée est de l'ordre de 2 à $2,5 \mathrm{~g}$, ce qui équivaut approximativement au tiers de l'azote soluble ingéré dans un aliment contenant une farine de poisson. L'évacuation rapide précoce d'une partie de l'azote soluble du poisson pourrait donc rendre compte de l'effet de la source protéique à l'égard de l'évacuation gastrique de l'azote total. De surcroît, les effets de la source de protéines à l'égard de l'évacuation de l'azote pourraient encore résulter au moins pour partie, d'une modification du niveau des apports endogènes par les sécrétions digestives, sous l"influence de l'appétibilité du régime (effets psychiques) ou de la composition particulière des protéines ingérées. On peut enfin noter que la nature des deux constituants principaux du régime (amidon, protéines) affecte l'évacuation gastrique de la matière sèche, alors que seule la source de protéines exerce un effet sur l'évacuation de la matière frâiche. Cette particularité pourrait encore traduire une importante différence des volumes secrétoires lors d'ingestion de régimes présentés sous une même dilution, mais comportant selon le cas farine de poisson ou gluten de blé, d'appétibilité fort différente. L'examen des données concer- 
nant la matière fraîche relativement à la matière sèche dans le tableau 3 suggère en effet un apport d'eau endogène nettement plus important dans le cas de la farine de poisson.

Un troisième effet indépendant identifié par l'analyse multidimensionnelle est "l'effet porc». Cette influence de l'animal, en tant qu'individu au sein de l'échantillon d'animaux constituant la population expérimentale, s'exerce significativement à l'égard de l'évacuation de la matière sèche et de l'azote mais ne concerne ni la matière frầche, ni l'amidon. Ce dernier point est relativement surprenant dans la mesure où il a déjà été constaté (CuBER, LAPlace \& Villiers, 1980) l'étroite association de l'évacuation de la matière sèche et de celle de l'amidon qui représente environ 70 p. 100 de l'ingéré sec. Cela étant, l'existence même d'un tel effet de l'individu conduit à s'interroger sur la taille optimum de l'échantillon d'animaux, nécessaire compte tenu de la variabilité des phénomènes étudiés. Dans ce domaine, les travaux habituellement réalisés n'impliquent que des nombres fort réduits d'animaux en raison de la nature des interventions chirurgicales préalables, de la difficulté d'entretien et d'utilisation de ces préparations chez le sujet éveillé en situation chronique et globalement de la charge que représentent de telles expérimentations. S'y ajoutent généralement l'absence de dispositif expérimental rigoureux et l'insuffisance des méthodes statistiques utilisées pour comparer les effets de régimes différant par plusieurs facteurs autres que ceux étudiés. Aussi, face à l'analyse des processus d'évacuation gastrique, la mise en cuvre d'un dispositif factoriel $2 \times 2$ complet et équilibré, impliquant des porcs fistulés, constitue une situation d'analyse hautement favorable. Dans ce contexte, les 12 porcs utilisés et affectés au hasard aux 4 combinaisons du plan factoriel doivent être considérés comme très homogènes : provenance d'un même élevage, mêmes antécédents, poids vifs très voisins (coefficient de variation 3,93 p. 100). L'existence de l'effet porc semble donc bien correspondre à une réalité physiologique et non à une insuffisance du dispositif expérimental.

Dans le cas de la matière sèche, le calcul des coefficients de variation des quantités résiduelles montre que ceux-ci sont compris entre 4 et 12 p. 100 pour les divers régimes aux temps $30 \mathrm{mn}, 1 \mathrm{~h}$ et $2 \mathrm{~h}$. Au temps $4 \mathrm{~h}$, ces coefficients sont compris entre 7 et 16 p. 100 et ils atteignent 24 à 43 p. 100 selon les régimes au temps 7 h. Cette divergence croissante des résultats au fil du déroulement de l'évacuation de la matière sèche est sans équivoque responsable de l'effet porc identifié par l'analyse multidimensionnelle, comme par les analyses de variance séparées pour les temps $4 \mathrm{~h}$ et $7 \mathrm{~h}$. On peut du reste souligner que les analyses de variance pour chaque instant d'observation reconnaissent aussi un effet porc au seul temps $7 \mathrm{~h}$ pour la matière fraîche et l'amidon. Il y a donc là un phénomène général, plus particulièrement important pour la matière sèche, qui traduit l'originalité du décours de l'évacuation gastrique de chaque animal à partir de la quatrième heure.

Pour ce qui concerne l'azote, l'effet porc identifié par l'analyse multidimensionnelle est d'une importance plus considérable encore puisque retrouvé à tous les temps de collecte par les analyses de variance réalisées séparément pour chaque instant d'observation. Cette permanence de l'effet porc correspond à l'existence, pour tous les temps de collecte, de coefficients de variation élevés (de l'ordre de 15 p. 100 pour le régime blé-poisson et 30 p. 100 pour les 3 autres régimes) des quantités absolues ou relatives d'azote résiduel dans l'estomac. La question se pose donc de savoir dans quelle mesure cette variabilité particulière de l'évacuation de l'azote entre porcs, à tous les instants d'observation, peut résulter des variations individuelles des apports 
endogènes : desquamation de la cavité buccale à l'estomac, apports liés aux sécrétions salivaire et gastrique diversement stimulées selon la réponse de chaque individu aux caractéristiques physico-chimiques et à l'appétibilité du régime.

\section{2) Les interactions entre facteurs}

Les diverses hypothèses ou interprétations exposées plus haut doivent cependant être nuancées, voire révisées, dans la mesure où l'analyse multidimensionnelle appliquée aux résultats concernant les régimes azotés met également en évidence des interactions significatives entre facteurs.

Ainsi une interaction entre nature de lamidon et source de protéines est-elle constatée pour toutes les variables contrôlées. Toute tentative visant à exprimer, sur la base des effets indépendants, des conclusions telles que «l'évacuation de la matière sèche (par exemple) est plus rapide lors d'ingestion du régime à base de l'amidon $A_{1}$ que lors d'ingestion du régime à base de l'amidon $A_{\text {.2 }} \gg$ ou encore «... est plus lente lors d'ingestion du régime comportant la protéine $P_{1}$ que lors d'ingestion du régime comportant la protéine $P_{2}$ ", ne peut donc qu'aboutir à une conclusion erronnée dans son caractère apparenıment généralisable. Les critiques formulées précédemment à l'égard de la grande complexité des régimes utilisés par divers auteurs, et de la difficulté qu'il y a dans de tels cas à maîtriser tous les facteurs de variation, restent valables. Mais, il apparaît aussi que, même pour des régimes ne comportant qu'un nombre très réduit de constituants, soit un amidon et une source azotée (inévitablement associés dans tout régime destiné au porc), il n'est pas possible d'aboutir à une claire définition de l'influence de l'un ou l'autre de ces deux constituants. En d'autres termes, toute hiérarchisation des amidons quant à leurs effets sur l'évacuation gastrique sera nécessairement remise en cause par la substitution d'une source protéique à une autre, et réciproquement. Cela signifie aussi que toute étude comparative des effets de 2 à $n$ amidons ingérés isolément, sans apport simultané d'aucun autre constituant, n'autorise aucune prévision de leurs influences respectives dans le cas d'association à une même source protéique et a fortiori à diverses sources de protéines alimentaires.

Une interaction significative entre la nature de l'amidon et le porc qui ingère cet amidon, est également constatée pour toutes les variables contrôlées. Ceci revient à dire que, abstraction faite des conséquences de l'interaction amidon-protéines, tous les animaux ne répondent pas de la même façon à l'ingestion d'un même amidon. Cette constatation est renforcée, en ce qui concerne l'évacuation gastrique de l'amidon et de lazote par l'existence d'une interaction triple (amidon - protéine - porc). Celle-ci signifie que chaque animal fournira un profil particulier d'évacuation gastrique de lamidon ou de l'azote à la suite de l'ingestion d'un aliment composé d'un couple donné amidon-protéines.

Enfin, l'évacuation gastrique de l'azote s'avère soumise à une interaction significative entre la source de protéines et le porc qui consomme ces protéines. Ainsi à la variabilité particulière de l'évacuation de l'azote telle qu'elle résulte de l'effet porc indépendant, qui pourrait refléter des différences d'animal à animal du niveau basal des apports endogènes, s'ajoute bien une différence entre porcs de leur réaction face à telle protéine. Cette différence pourrait être le fait d'un degré variable de la stimulation des apports secrétoires induite par l'ingestion d'une même protéine. 


\section{3) Influence du temps}

La non indépendance des données recueillies n'autorise pas l'étude du facteur temps dans le cadre de l'analyse de variance. Un intérêt de la décomposition polynomiale des effets, permettant en définitive d'assimiler valablement les divers profils d'évacuation gastrique à des polynômes de degré 2 (matière fraîche) ou 3 (autres variables), est précisément, du fait même du degré significatif de la régression du volume évacué en fonction du temps, de démontrer qu'il existe effectivement une influence du temps sur les observations. Cette affirmation d'un effet temps indépendant peut paraître d'évidence, l'évacuation de l'estomac signifiant implicitement que la quantité évacuée cumulée croît progressivement au cours des heures postprandiales. Mais un apport supplémentaire découle de l'ajustement des observations par des polynômes du degré significatif déterminé par la décomposition polynomiale des effets, et de la comparaison de ces polynômes (ou courbes de la figure 4).

En effet, pour les variables matière fraîche, matière sèche et amidon, les comparaisons prenant en compte globalement les 4 courbes (correspondant aux 4 combinaisons amidon-protéine) conduisent à rejeter l'égalité comme le parallélisme. Ces différences traduisent pour les 3 variables concernées une interaction entre l'effet du temps et les effets mis en évidence par l'analyse de variance. Dans le cas de l'évacuation de l'azote le parallélisme est globalement recevable, en l'absence d'égalité, ce qui revient à dire que seul le terme constant $\left(b_{0}\right)$ du polynôme est soumis aux effets expérimentaux en dehors de tout effet du temps.

Pour tout expérimentateur se bornant à observer les données expérimentales, telles qu'elles sont présentées dans la première partie de l'exposé des résultats, il paraît presque évident qu'existent des interférences entre les facteurs dont on ne peut aisément caractériser un effet simple. Il lui apparaîtra aussi que les effets, qu'ils soient ou non le produit d'une interaction entre facteurs, évoluent au cours du temps. Ainsi, il constatera par exemple que pour les régimes à base d'amidon de maïs, les quantités résiduelles de matière fraîche sont plus importantes lors d'association au poisson alors que pour des régimes à base d'amidon de blé, elles sont, au-delà de la troisième heure postprandiale, supérieures lors d'association au gluten. De même, des quantités résiduelles de matière sèche plus importantes sont mesurées pour le couple maïs-poisson que pour le couple maïs-gluten pendant les 3 premières heures postprandiales. De la même façon, les quantités résiduelles d'amidon sont, pour des régimes à base d'amidon de maïs, plus importantes au cours des 2 premières heures postprandiales lors d'association au poisson, alors que pour les régimes à base d'amidon de blé, ces quantités résiduelles d'amidon sont, au-delà de la troisième heure postprandiale, supérieures lors d'association au gluten. En bref, toute tentative d'expression d'un effet observé doit être affectée d'une restriction quant aux limites temporelles de sa validité. Cette évidente difficulté ressort de l'existence démontrée plus haut d'une interaction entre le facteur temps et les autres facteurs expérimentaux, au moins pour la matière fraîche, la matière sèche et l'amidon.

Au total, on peut donc retenir dans un premier bilan : (i) que les variables contrôlées sont soumises à l'influence de 4 facteurs au moins, tels qu'ils sont identifiables dans le schéma expérimental : nature de l'amidon, source de protéines, animal concerné, et temps écoulé depuis le repas; (ii) que ces facteurs interagissent entre eux : amidonprotéine et amidon-porc (pour toutes les variables), protéine-porc (pour l'azote seulement), amidon-protéine-porc (pour l'amidon et l'azote évacués), temps et tous les 
autres facteurs identifiés (pour la matière fraîche, la matière sèche et l'amidon); (iii) que dans tous ces cas les divers termes du polynôme représentatif sont soumis aux effets et/ou interactions énumérés alors que, pour l'azote, seul le terme constant du polynôme est soumis à ces influences.

Deux sortes de questions se trouvent donc posées. En premier lieu, la signification physiologique des divers termes des polynômes est à établir, les coefficients représentant en quelque sorte l'expression mathématique de l'action de mécanismes fonctionnels dont la modification sous l'influence des effets identifiés conduit à produire tel ou tel profil particulier d'évacuation. En second lieu, la multiplicité des interactions enregistrées conduit à šinterroger sur la possibilité d'atteindre à une conclusion valable lors de la comparaison de l'évacuation gastrique de 2 régimes distincts. Sur ce dernier point une réponse pourra être apportée par le résultat des tests d'égalité et de parallélisme dans le cadre des comparaisons 2 à 2 des polynômes.

\section{B. - Evacuation gastrique selon la nature de l'amidon et des protéines des régimes azotés}

\section{1) Signification physiologique des coefficients polynomiaux}

Attribuer une signification physiologique aux coefficients polynomiaux est fort difficile dans la mesure où chacun d'eux exprime nécessairement de façon synthétique la résultante d'un ensemble de facteurs de contrôle, stimulants ou inhibiteurs de l'évacuation gastrique, qui s'exercent à des degrés divers au cours du temps et selon laliment ingéré.

Un ajustement moyen par régression polynomiale avait déjà été utilisé (LAPLACE \& Tomassone, 1970) pour comparer les volumes de matière fraîche recueillis au niveau d'une fistule duodénale proximale chez des porcs ingérant un aliment standard (céréales-soja). Sur la base de ces volumes de matière fraîche, l'évacuation gastrique avait été représentée par des polynômes de degré 3. Dans le présent travail, le degré significatif des polynômes exprimant les poids de matière fraîche évacuée, déterminé par la décomposition polynomiale des effets, est le degré 2. De surcroît, le coefficient $b_{0}$ est ici presque constamment positif alors qu il était généralement négatif dans létude antérieure (LAplace \& Tomassone, 1970). Toutefois, les conditions expérimentales utilisées ici sont différentes (mesure de quantités résiduelles au niveau d'une fistule gastrique ; régimes semi-purifiés; expression en poids de contenus frais).

En dépit de ce rapprochement difficile, le principe retenu pour interpréter d'un point de vue fonctionnel les coefficients polynomiaux reste utilisable. Ainsi le terme $b_{0}$, qui rend compte de l'ordonnée à l'origine de la courbe des Y (variable mesurée) en fonction du temps, traduit l'état de «disponibilité »du système, celle-ci étant d'autant plus grande que le terme $b_{0}$ croît du négatif vers le positif. A une positivité accrue devrait correspondre une plus grande précocité de l'évacuation. Le terme $b_{1}$ correspond à la vitesse initiale du phénomène d'évacuation et exprime donc globalement la résultante des divers effets excitomoteurs liés à l'ingestion du repas et à la présence des matériaux alimentaires dans l'estomac. Ce coefficient $b_{1}$ devrait être d'autant plus positif que les effets excitomoteurs sont globalement plus intenses. Le terme $b_{2}$, qui s'avère constamment négatif, exprime la décélération du processus d'évacuation sous l'influence de l'ensemble des effets inhibiteurs nerveux et humoraux mis en jeu au niveau du duodénum par l'arrivée des digesta évacués. Selon les carac- 
téristiques physico-chimiques de ces derniers, le coefficient $b_{2}$ devrait être d'autant plus négatif que sont globalement plus actifs les facteurs assurant le freinage de l'évacuation. Enfin, le terme $b_{3}$ rend compte de l'assymétrie de la courbe de part et d'autre de son point d'inflexion; ce terme devrait être d'autant plus positif que l'assymétrie est plus forte. Le coefficient $b_{3}$ exprimant aussi une accélération, il correspond à une relance du processus d'évacuation préalablement freiné sous l'influence des facteurs englobés dans le terme $b_{g}$. L'assymétrie de part et d'autre du point d'inflexion exprime donc l'évolution de la dominance relative des facteurs inhibiteurs sur les facteurs stimulants et réciproquement. Au total $b_{3}$ est l'expression de l'équilibre des facteurs de contrôle antagonistes assurant une régulation de l'évacuation gastrique. Ces divers éléments ne constituent qu'une généralisation de l'interprétation proposée antérieurement (LAPLACE \& TOMASSONE, 1970) et raisonnée alors principalement en termes volumétriques de réplétion digestive.

\section{2) Caractéristiques de l'évacuation gastrique selon le régime : étude analytique}

Sur ces bases, les effets des régimes expérimentaux peuvent être comparés, de façon analytique, en les classant en fonction de la valeur des coefficients polynomiaux des ajustements moyens pour telle ou telle variable mesurée. Ce classement simple permet donc de comparer leurs effets à l'égard de la précocité de l'évacuation $\left(b_{0}\right)$, de son intensité initiale $\left(b_{1}\right)$, de limportance de son freinage $\left(b_{2}\right)$ et de sa relance ultérieure $\left(b_{3}\right)$.

Globalement, et compte tenu de la signification accordée aux coefficients polynomiaux, on peut noter que l'évacuation de la matière fraîche se présente comme un phénomène relativement régulier s'amortissant au cours du temps. Ce décours sans point d'inflexion doit être relié au fait que les apports d'eau d'origine secrétoire (salivaire et gastrique) sont les plus importants en période post prandiale rapprochée (phases céphalique et gastrique de sécrétion) mais que l'évacuation des liquides est toujours rapide et précoce (Laplace \& Tomassone, 1970). A l'opposé, l'évacuation des matériaux de la phase solide est beaucoup plus longue et d'intensité variable selon limportance respective des facteurs stimulants ou inhibiteurs. Il en résulte les profils du troisième degré retenus pour la matière sèche, l'amidon et l'azote. Ces courbes comportant un point d'inflexion correspondent à l'observation ancienne du fait que l'évacuation gastrique se présente comme un phénomène «biphasique »: deux périodes d'évacuation relativement intense, séparées par une période de moindre débit (AufFray, Martinet \& Rerat, 1967).

La comparaison analytique des effets des régimes sur les caractéristiques de lévacuation (précocité, intensité...) conduit à noter pour la matière fraîche que l'évacuation est plus précoce pour les aliments contenant du gluten que pour ceux qui comportent de la farine de poisson. L'évacuation initialement la plus intense fait aussi lobjet du plus fort freinage ultérieur (cas du régime maïs-poisson) et inversement (faible intensité initiale et faible décélération dans le cas du régime blé-gluten). Un tel phénomène a déjà été rencontré pour l'évacuation de la matière fraîche de régimes à base d'amidon cru ou extrudé de pomme de terre (BorgidA \& LAPLACE, 1977) : évacuation initialement rapide mais fortement freinée ultérieurement pour l'amidon le moins hydrolysable; évacuation plus régulière pour l'amidon le plus rapidement hydrolysé.

En ce qui concerne l'évacuation de la matière sèche, il est remarquable que la vitesse initiale soit plus grande, la décélération plus intense et l'assymétrie plus forte, 
pour les régimes contenant du gluten que pour ceux qui comportent de la farine de poisson (particularités évidentes à l'examen de la figure $4 \mathrm{~B}$ ). La vitesse initiale élevée d'évacuation de la matière sèche des régimes gluten pourrait être reliée à la lenteur de mise en jeu de certains facteurs inhibiteurs d'origine duodénale en raison de la lenteur d'hydrolyse des protéines du gluten de blé. Il s'agit, en effet, d'une protéine de configuration complexe du fait d'une teneur élevée en proline comparativement à une farine de poisson (Henry, Pion \& Rerat, 1976). Que l'inhibition se développe lentement peut également expliquer qu'elle n'en soit que plus intense dans la phase de décélération et qu'il en résulte enfin une assymétrie forte.

Ce phénomène de balancement selon l'importance relative des facteurs stimulants et des facteurs inhibiteurs est encore retrouvé dans le cas de l'évacuation de l'amidon. Schématiquement, on constate que celle-ci est plus précoce, mais initialement moins rapide, soumise à une décélération plus faible et à une relance moindre, dans le cas des régimes maïs-gluten et blé-poisson par rapport aux régimes blé-gluten et maïspoisson. Enfin, dans le cas de l'évacuation de l'azote, le classement des régimes est rigoureusement identique pour les coefficients $b_{1}$ (du plus au moins positif), $b_{2}$ (du plus au moins négatif) et $b_{33}$ (du plus au moins positif). Par contre, un classement différent peut être établi sur la base des coefficients $b_{0}$, particularité qui correspond au fait que seul ce dernier coefficient est soumis aux effets expérimentaux en dehors de toute interaction avec le temps.

Au-delà des différences que révèle une telle comparaison analytique des effets relatifs des divers régimes les uns par rapport aux autres sur les caractéristiques du profil d'évacuation, une sorte de loi générale de déroulement du processus tend à se dégager. Il apparaît en effet qu'une évacuation se déroulant initialement à une vitesse élevée conduira nécessairement à la mise en jeu massive des facteurs inhibiteurs d'où résultera une forte décélération. Cette première relation signifie une certaine proportionnalité de l'inhibition d'origine duodénale à la «quantité de stimulation »liée à la quantité et/ou qualité des matériaux déversés dans le duodénum. A cette phase de freinage succède une nouvelle phase rapide correspondant à l'accélération $\left(b_{3}\right)$ d'autant plus forte que le freinage aura été plus intense. Cette seconde relation semble aussi stable que la première. Globalement, plus ces phénomènes de balancement seront importants, plus le profil d'évacuation sera heurté (ou l'assymétrie plus forte). Tel devrait être le cas pour les substrats dont l'attaque enzymatique est lente et dont les produits d'hydrolyse tardivement libérés n'entraînent d'inhibition rétrograde qu'après une période d'évacuation rapide peu contrôlée. A l'inverse les substrats rapidement hydrolysés pour lesquels le contrôle inhibiteur peut intervenir, même modérément, de façon précoce, conduiront à un profil d'évacuation plus régulier ( $b_{1}$ moins positif, $b_{2}$ moins négatif), et à une moindre assymétrie ( $b_{3}$ moins positif).

\section{3) Profils d'évacuation gastrique selon le régime}

Si la comparaison analytique des caractéristiques de l'évacuation pour tel ou tel régime est particulièrement intéressante au plan des mécanismes physiologiques sous jacents, la comparaison des profils par les tests d'égalité et de parallélisme permet d'approcher la résultante des effets qui importe au plan nutritionnel. En effet, la mise en jeu de degrés fort différents de stimulation et d“inhibition selon les régimes n̈implique pas nécessairement, dans la mesure où il y a une certaine proportionnalité entre les caractéristiques exprimées par les coefficients du polynôme, que la résultante soit 
différente. En d'autres termes, un même profil d'évacuation gastrique pourrait fort bien être assuré avec des niveaux différents des divers facteurs de contrôle d'ordre nerveux et humoral. Cela signifierait par exemple et de façon très schématique que, après ingestion d'un régime $A$, l'existence de taux de telle hormone réputée inhibitrice plus élevés qu'après ingestion d'un régime $B$, ne saurait impliquer une moindre évacuation gastrique du régime $\mathrm{A}$ dans la mesure où cette influence négative peut être contrebalancée par un niveau supérieur des facteurs stimulants dont l'effet est exprimé par $b_{1}$ et $b_{3}$.

Le résultat global de l'évolution au cours du temps de l'équilibre entre les divers facteurs de contrôle est fourni par les profils polynomiaux de la figure 4. Les résultats des tests (égalité, parallélisme) illustrent les propositions avancées ci-dessus. Un premier exemple est fourni par l'évacuation de la matière sèche des régimes blé-gluten et maïs-gluten. Pour ces deux cas les valeurs de $b_{0}$ sont largement différentes, mais les valeurs de $b_{1}, b_{2}$ et $b_{3}$ sont voisines. Ces caractéristiques font que les profils d'évacuation de la matière sèche pour ces deux régimes sont parallèles mais non identiques du fait de la différence d'ordonnée à l'origine. Un second exemple peut être emprunté à l'évacuation de l'amidon après ingestion des régimes blé-gluten et blé-poisson. Les ordonnées à l'origine sont cette fois assez voisines, mais les valeurs de $b_{1}, b_{2}$ et $b_{3}$ pour ces régimes sont largement différentes; pourtant les profils d'évacuation de l'amidon pour ces deux régimes sont parallèles et égaux.

Ce qui précède amène donc à affirmer que, à partir des mêmes mesures directes de l'évacuation de l'estomac, les effets de la composition du régime peuvent être analysés sous deux aspects : l'un concerne les modifications des divers facteurs de contrôle et présente un intérêt particulier pour le physiologiste ; l'autre est relatif au résultat global de ces effets, c'est-à-dire au décours de l'évacuation au cours du temps, aspect plus particulièrement intéressant pour le nutritionniste. On peut également en conclure que des différences importantes dans l'intensité de mise en jeu des divers facteurs de contrôle peuvent certes répondre de profils d'évacuation différents, mais peuvent aussi fort bien conduire à des profils d'évacuation tout à fait semblables. Par conséquent, aux déductions précédemment formulées quant à l'impossibilité d'assurer une prévision des effets de tel constituant alimentaire dans diverses conditions d'association, à partir des effets observés dans une situation donnée, il convient d'en ajouter une autre : l'étude directe des effets de tel constituant alimentaire sur les divers éléments du contrôle de l'évacuation (activités nerveuses; libération d'hormones gastrointestinales) n'autorise pas non plus une prévision du résultat global en l'état actuel des possibilités d'investigation simultanée de ces divers éléments.

Au total, pour la comparaison des profils d'évacuation gastrique lors d'ingestion de régimes différents, l'ajustement polynomial des données, tel qu'il est ici utilisé pour l'analyse mathématique des phénomènes et complété par les tests d'égalité et de parallélisme, s'avère un outil descriptif puissant et fiable, rendant caducs les tests statistiques couramment appliqués aux quantités moyennes évacuées à tel ou tel temps postprandial. En définitive, tous les effets expérimentaux étudiés par l'analyse multidimensionnelle étant pris en compte, y compris dans leurs interactions, les analogies ou différences d'évacuation gastrique de la matière fraîche, de la matière sèche, de l'amidon et de l'azote au cours des 7 heures après ingestion de l'une ou l'autre des quatre combinaisons d'un amidon purifié (maïs-blé) et d'une source de protéines (poisson-gluten) sont intégralement exprimées dans la figure 4. 


\section{4) Etude des effets expérimentaux dans le cas des régimes protéiprives}

Les résultats de l'analyse multidimensionnelle globale portant sur les quantités évacuées après ingestion des régimes protéiprives (tableau 7), c'est-à-dire d'amidon de maïs ou d'amidon de blé purifiés, peuvent être examinés à la fois en eux mêmes et par rapport aux résultats relatifs aux régimes azotés (tableau 6).

En ce qui concerne l'évacuation de la matière fraîche, on retrouve en régime protéiprive une interaction amidon-porc ; il s'y ajoute cependant un effet porc indépendant qui n'était significatif en régime azoté qu'à l'instant d'observation $7 \mathrm{~h}$. Dans le cas de l'azote on retrouve les effets de la nature de l'amidon et du porc en l'absence toutefois d'interaction entre ces deux facteurs. Néanmoins, pour ces deux variables, matière fraîche et azote, les résultats des deux analyses multidimensionnelles (régimes azotés vs protéiprives) sont cohérents. Tel n'est pas le cas pour la matière sèche et l'amidon puisque dans le cas des régimes protéiprives, seul est enregistré un effet porc indépendant à l'exclusion de tout effet de la nature de l'amidon qui représente pourtant 90 p. 100 de l'aliment ingéré. Cette absence d'effet de la nature de l'amidon lorsqu'il est consommé sans protéines, alors qu'elle est responsable de différences dans un régime azoté, ne fait que confirmer la difficulté de prévision des effets d'un constituant du régime à partir d'observations conduites dans une situation particulière.

Cela étant, la comparaison des quantités résiduelles dans l'estomac, à un même instant d'observation, pour des régimes protéiprives par rapport aux régimes azotés conduit à des conclusions cohérentes avec diverses données bibliographiques comme avec ce qui a été précédemment déduit de l'étude analytique des effets des régimes utilisés sur l'évacuation gastrique. L'observation des valeurs moyennes des mesures révèle par exemple que la quantité résiduelle de matière sèche au stade $30 \mathrm{mn}$ est plus faible avec le gluten qu'avec le poisson, cet écart s'annulant au-delà de la deuxième heure pour s'inverser ensuite. On note par ailleurs que, globalement, la matière sèche des régimes protéiprives est évacuée plus rapidement que celle des régimes azotés au cours des premières heures postprandiales, en accord avec divers auteurs (RERAT \& LOUGNON, 1963 ; Zebrowska \& BURACZEWSKA, 1972 ; ZebrowsKa et al., 1975). Il est cependant remarquable que, durant les deux premières heures postprandiales, l'évacuation de la matière sèche des régimes gluten est analogue à celle constatée pour les régimes protéiprives (indifféremment blé ou maïs, en l'absence d'effet significatif de la nature de l'amidon sur l'évacuation de la matière sèche des régimes protéiprives). Cette analogie temporaire (dans le sens d'une relative rapidité d'évacuation de la matière sèche) pourrait fort bien traduire une même absence de certains effets inhibiteurs, soit du fait du défaut de protéines dans le régime (cas protéiprive), soit par suite de la lenteur d'hydrolyse des protéines de configuration complexe (cas du gluten).

De façon corollaire, pour des temps postprandiaux plus importants, ces mêmes effets inhibiteurs font toujours défaut dans le cas des régimes protéiprives, alors qu'ils ont pu s'exercer dans le cas des régimes gluten. Ceci est en accord avec le fait qu'au temps $7 \mathrm{~h}$ une plus forte quantité de matière sèche a été évacuée pour les régimes protéiprives que pour les régimes gluten. Une telle lenteur relative d'évacuation de la matière sèche est également observée au bout de $8 \mathrm{~h}$ par ZeBrowska (1973 a) pour un régime comportant du gluten. Cela étant, on notera aussi que, parmi les régimes testés par ZEBrowska (1973 a) la matière sèche de ceux qui comportent de la caséine ou du gluten est évacuée moins rapidement que pour des régimes comportant du soja cru ou cuit ou de la féverole. Or, caséine et gluten présentent des taux de proline 
comparables et nettement plus élevés que ceux des autres sources protéiques utilisées (ZEBROWSKA, 1973 b).

L'absence d'influence significative de la nature de l'amidon ingéré sur l'évacuation de l'amidon en situation protéiprive fait que l'on peut aussi, comme pour la matière sèche, observer globalement une évacuation plus rapide de l'amidon pour les régimes protéiprives que pour les régimes azotés au stade $2 \mathrm{~h}$.

En ce qui concerne l'évacuation gastrique de lazote, un effet significatif de la nature de l'amidon constituant le régime protéiprive est mis en évidence par l'analyse de variance. Ainsi l'azote endogène serait évacué plus rapidement - ou produit en quantité plus faible - lors d'ingestion de l'aliment protéiprive maïs par rapport au régime protéiprive blé. La différence est minime lorsqu'on considère les quantités indiquées dans le tableau 4. Mais cette particularité signifie que la production d'azote endogène, soumise à un effet porc significatif (régimes azotés et protéiprives) et à l'interaction protéine-porc dans le cas des régimes azotés, est également affectée par la nature de l'amidon lorsque celui-ci est ingéré seul.

\section{5) Evacuation gastrique et utilisation digestive des aliments}

Il a été indiqué initialement que parmi les facteurs de variation potentiels de l'utilisation digestive des aliments chez le porc, l'évacuation gastrique pourrait jouer un rôle non négligeable dans la mesure où elle contrôle l'arrivée des matériaux dans l'intestin grêle où s'effectuent pour l'essentiel hydrolyses enzymatiques et absorption des nutriments. Cette idée a priori doit être confrontée aux résultats enregistrés pour l'évacuation de l'amidon et de l'azote. Les aliments du porc sont en effet constitués pour l'essentiel de céréales (sources d'amidon) et de protéines (sources d'azote).

Dans cette perspective, et sur la base des comparaisons de profils polynomiaux d'évacuation (figure 4) on constate que l'évacuation gastrique de l'amidon répond à un même profil moyen pour les régimes blé-gluten et blé-poisson, le régime maïs-gluten n'en différant que par l'ordonnée à l'origine. Ceci signifie que pour ces trois couples amidon-protéines, à défaut d'une identité quantitative dans le cas du maïs-gluten, la cinétique de mise à disposition de l'amidon pour les processus d'hydrolyse et d'absorption sera la même. Cela est encore plus vrai pour l'évacuation gastrique de l'azote puisque les 4 profils sont similaires, trois d'entre eux répondant de surcroît à un même profil moyen. Par conséquent, à défaut d'identité quantitative pour l'association blé-gluten, la cinétique de mise à disposition des matières azotées sera la même pour les 4 régimes testés.

Ces constatations, pour limitées qu'elles soient aux cas considérés, n'en sont pas moins cohérentes avec la conclusion retenue au terme d'un précédent travail (Cuber, Laplace \& Villiers, 1980) : l'évacuation gastrique de l'amidon n'est pas le facteur limitant contrôlant la cinétique d'apparition des sucres réducteurs dans la veine porte. Les différences qui peuvent être constatées dans cette cinétique d'absorption sont plus probablement à relier à des différences de vitesse d'hydrolyse des divers amidons (Cuber, Laplace \& Villiers, 1980).

Par ailleurs, l'analogie ou identité des cinétiques d'arrivée des matières azotées dans l'intestin grêle ne fournit aucune base d'explication de la différence considérable de gain de poids vif constatée entre porcs recevant les régimes poisson et porcs recevant les régimes gluten. Là encore, c'est au-delà de l'estomac que se déterminent les 
différences. Il apparaît en effet, pour les 4 régimes, des différences de digestibilité apparente de l'azote mesurée à l'extrémité distale de l'intestin grêle (DARCY, LAPLACE \& Villiers, 1981), tant du fait de la nature de l'amidon que de celui de la source de protéines avec également interaction entre ces facteurs. Par ailleurs, la croissance médiocre des porcs recevant les régimes gluten a pu être attribuée plus particulièrement à une très mauvaise utilisation métabolique de l'azote dans ce cas (DARCY \& LAPLACE, 1981).

Au total, sans qu'il soit possible a priori de généraliser cette conclusion, la cinétique d'évacuation gastrique ne permet guère d'expliquer une part des variations dans l'utilisation digestive des aliments. De plus, l'usage des régimes purifiés ne permet pas de définir un certain nombre de principes autorisant une prévision des effets sur lévacuation gastrique puisque, de toutes façons, toute nouvelle association de constituants alimentaires exige une réévaluation des effets. L'intérêt des régimes purifiés s'en trouve donc réduit dans une perspective d'étude de nutrition animale; il reste par contre très grand pour approcher les phénomènes physiologiques sous jacents qui concourent à produire tel ou tel profil d'évacuation gastrique.

\section{Conclusions}

La méthodologie d'analyse mathématique, mise en œuvre pour l'étude comparative de l'évacuation gastrique de divers régimes, et fondée sur l'analyse multidimensionnelle, la décomposition polynomiale des effets du modèle, et la comparaison de courbes répondant à un ajustement polynomial des données, s'avère être un outil de travail particulièrement efficace. Il autorise en effet l'analyse des effets mis en jeu y compris l'influence du temps, celle des modifications globales qui en découlent au niveau des facteurs de contrôle de l'évacuation, et enfin la comparaison des profils d'évacuation résultants.

11 apparaît que les variables contrôlées sont soumises à l'influence de la nature de lamidon, de la source de protéines et de l'animal utilisé ; mais il existe aussi des interactions telles entre ces diverses influences, que les effets d'un amidon ou d'une protéine évalués dans une situation donnée n’autorisent pas une prévision de leurs effets respectifs dans d'autres associations. De surcroît, il existe des interactions des effets amidon ou protéine avec l'effet du porc, en particulier à l'égard de l'azote évacué par l'estomac, révélant l'originalité de chaque individu non seulement quant à sa production basale d'azote endogène, mais quant à sa réponse à la nature des matériaux alimentaires qu'il ingère.

Les profils d'évacuation de la matière fraîche peuvent être valablement ajustés à des polynômes de degré 2 . Ceux de la matière sèche, de l'amidon et de l'azote sont identifiables à des polynômes de degré 3 , ce qui démontre l'influence du temps sur ces observations. Les effets expérimentaux concernent tous les termes du polynôme représentatif pour la matière sèche et l'amidon; ils n'affectent que le seul terme constant pour l'azote.

Les divers termes des polynômes représentatifs correspondent à un ensemble de phénomènes physiologiques constituant les facteurs de contrôle stimulants ou inhibiteurs de l'évacuation. La valeur de ces termes rend compte du degré de mise en jeu 
de ces divers facteurs et traduit la résultante de leurs effets en caractéristiques de vitesse, de décélération puis de relance du processus d'évacuation. Cependant, des niveaux très différents d'activation des facteurs de contrôle, observés pour des régimes distincts, peuvent fort bien conduire à un même décours de l'évacuation gastrique.

$\mathrm{Au}$ total, pour les régimes utilisés et en dépit de la complexité des effets en cause, une évacuation similaire de la matière sèche est observée pour les régimes contenant de la farine de poisson quel que soit l'amidon, tandis que des profils semblables (mais différents des précédents) sont observés pour les régimes contenant du gluten de blé, quel que soit l'amidon. En ce qui concerne l'évacuation de l'amidon, des profils semblables voire égaux sont constatés à l'exception du cas du régime maïspoisson cependant que tous les profils d'évacuation de l'azote sont similaires. Enfin, globalement, le rythme d'évacuation gastrique n'apparaît pas comme un facteur de contrôle limitant de l'utilisation digestive des aliments.

Accepté pour publication en mars 1981.

\section{Summary}

Effect of the kind of starch (wheat or maize) and proteins (fish or gluten) on control factors and pattern of the gastric emptying of a semi-purified diet in the pig. Application of multivariate analysis and polynomial regression

Twelve Large White pigs, weighing $51.3 \mathrm{~kg}$ on an average, were fitted with permanent gastric cannulas to measure the residual gastric contents at various times after a test meal (30 mn, 1, 2, 4 and $7 \mathrm{~h}$ ). Four diets including $16 \%$ crude protein and prepared according to a factorial combination of 2 purified starches (wheat or maize) and 2 protein sources (fish meal or wheat gluten) were offered (after a period of adaptation) in 15 test meals to each pig ( 3 tests per collection time; 3 pigs per diet). Parallel to that, 2 protein-free diets based on maize or wheat starch were given without previous adaptation, to allow 2 collections in each pig 2 hours after the meal and 2 collections in each pig 7 hours after the meal. Residual amounts of fresh matter, dry matter, starch and nitrogen in the stomach were measured.

A methodology based on a mathematical analysis was applied; it consisted in a multivariate analysis of variance to investigate the experimental effects (starch, protein, pig), a polynomial decomposition of the effects so as to determine the influence of time, and a comparison of gastric emptying curves resulting from a polynomial adjustment of the data. The coefficients of these polynomes were interpreted as physiological control factors.

The controlled variables were submitted to the influence of the kind of starch, protein source and animal used. However, there were also interactions such as the effects of one starch or one protein, estimated in a given situation, which did not allow any prediction of their respective effects in other associations. In addition, the interaction of the starch and/or protein effects with the pig effect, especially with respect of nitrogen, showed the originality or each individual not only in terms of basal production of endogenous nitrogen, but also in terms of response to the kind of dietary materials ingested. The influence of time on the different observations was evidenced for dry matter, starch and nitrogen. The polynomial coefficients were used to analyse the modifications of the degree of activation of the factors controlling the gastric emptying. But, the balance between antagonist control factors, whose activation was very variable, could result in similar or even equal emptying patterns.

Comparisons of the gastric emptying curves obtained for each variable tested are fully reported in figure 4, for all diets used. Moreover, the gastric emptying does not seem to be a limiting factor of the digestive utilization of food. 


\section{Références bibliographiques}

Auffray P., Martinet J., Rerat A., 1967. Quelques aspects du transit gastrointestinal chez le Porc. Ann. Biol. anim. Biochim. Biophys., 7, 261-279.

Borgida L.P., LAPlace J.P., 1977. Evacuation gastrique comparée de régimes à base de fécule de pomme de terre crue ou extrudée chez le Porc en croissance. Ann. Zootech., 26. $585-593$.

Cuber J.C., Laplace J.P., 1979. Evacuation gastrique de l'amidon et de l'azote d'un régime semi-purifié à base d'amidon de maïs chez le Porc. Ann. Zootech., 28, 173-184.

Cuber J.C., Laplace J.P., Villiers P.A., 1980. Fistulation de l'estomac et contenus gastriques résiduels après ingestion d'un régime semi-purifié à base d'amidon de maïs chez le Porc. Reprod. Nutr. Dev., 20, 1161-1172.

Darcy B., 1980. Contribution à l'étude de la digestion dans l'intestin grêle chez le Porc : Etude de l'interaction entre la nature de l'amidon et la source protéique du régime. Thèse Doct. Ing., Paris, 107 p.

Darcy B., Laplace J.P., 1981. Bilans nutritionnels chez le Porc selon la nature de l'amidon (blé ou maïs) et la source de protéines (poisson ou gluten). Ann. Zootech., 30, 63-76.

Darcy B., Laplace J.P., Villiers P.A., 1981. Digestion dans l'intestin grêle chez le Porc. 4) Cinétique de passage des digesta au niveau de la jonction ileo-caeco colique et bilans de la digestion selon la nature de l'amidon et la source de protéines alimentaires. Ann. Zootech., 30, 31-62.

Guilbot A., 1968. Structure de l'amidon. Effets de quelques traitements de technologie alimentaire, pp. 11-28. In : Lavollay J., Progrès en Chimie agricole et alimentaire, Hermann Ed., Paris.

Henry Y., Pion R., Rerat A., 1976. Protein supply for pigs and possibilities of reducing protein feeding standards. World Rev. anim. Prod., 12, 9-32.

Holmes J.H.G., Bayley H.S., HoRney F.D., 1974. Digestion of dry and high moisture maize diets in the stomach of the pig. Br. J. Nutr., 32, 639-646.

Ivan M., Farrell D.J., 1976. Nutritional evaluation of wheat. 5) Disappearance of components in digesta of pigs prepared with two re-entrant cannulae. Anim. Prod., 23, 111119.

Keys J.E., De Barthe J.V., 1974. Site and extent of carbohydrate, dry matter, energy and protein digestion and the rate of passage of grain diets in swine. J. anim. Sci., 39, 57-62.

KrishnaIAH P.R., 1969. Multivariate analysis of variance and related topics, pp. 55 à 168. In: Multivariate analysis, part II, 1 vol., Academic Press, New York.

Laplace J.P., Tomassone R., 1970. Evacuation gastro-duodénale chez le Porc; fistulation chronique par voie thoracique extrapleurale; recherche d'une technique d'analyse mathématique de l'évacuation. Ann. Zootech., 19, 303-332.

Low A.G., 1979. Studies on digestion and absorption in the intestines of growing pigs. 5) Measurements of the flow of nitrogen. Br. J. Nutr., 41, 137-146.

Low A.G., Partridge I.G., Sambrook I.E., 1978. Studies on digestion and absorption in the intestines of growing pigs. 2) Measurements of the flow of dry matter, ash and water. Br. J. Nutr., 39, 515-526.

PotThof R.F., Roy S.N., 1964. A generalized multivariate analysis of variance model useful especially for growth curve problems. Biometrika, 51, 313-326.

Rerat A., Lougnon J., 1963. Etudes sur le transit digestif chez le Porc. Ann. Biol. anim. Biochim. Biophys., 3 ( $\mathrm{N}^{\circ}$ hors série), 21-27.

Samвrook I.E., 1979. Studies on digestion and absorption in the intestines of growing pigs. 7) Measurements of the flow of total carbohydrate, total reducing substances and glucose. Br. J. Nutr., 42, 267-277. 
ZEBrowsKa T., 1973 a. Influence of dietary protein source on the rate of digestion in the small intestine of pigs. 1) Amount and composition of digesta. Rocz. Nauk. roln., 95 B, 115-133.

ZeBrowsKa T., $1973 \mathrm{~b}$. Influence of dietary protein source on the rate of digestion in the small intestine of pigs. 2) The rate of protein digestion and amino acids absorption. Rocz. Nauk. roln., $95 \mathrm{~B}, 135-155$.

Zebrowska T., BuraczewsKa L., 1972. Influence of dietary protein level on the rate of digestion in the small intestine of pigs. 1) Amount and composition of digesta (en polonais). Rocz. Nauk. roln., $94 \mathrm{~B}, 81-95$.

Zebrowska T., Buraczewska L., Buraczewski S., Horszczaruk F., 1975. Digestion and absorption in the small intestine of pigs. 1) Digestion and absorption of dry matter and nitrogen. Rocz. Nauk. roln., 96 B, 79-90. 